MATHEMATICS OF COMPUTATION

Volume 76, Number 260, October 2007, Pages 1895-1923

S 0025-5718(07)01987-4

Article electronically published on April 19, 2007

\title{
AN EXPONENTIALLY CONVERGENT ALGORITHM FOR NONLINEAR DIFFERENTIAL EQUATIONS IN BANACH SPACES
}

\author{
IVAN P. GAVRILYUK AND VOLODYMYR L. MAKAROV
}

\begin{abstract}
An exponentially convergent approximation to the solution of a nonlinear first order differential equation with an operator coefficient in Banach space is proposed. The algorithm is based on an equivalent Volterra integral equation including the operator exponential generated by the operator coefficient. The operator exponential is represented by a Dunford-Cauchy integral along a hyperbola enveloping the spectrum of the operator coefficient, and then the integrals involved are approximated using the Chebyshev interpolation and an appropriate Sinc quadrature. Numerical examples are given which confirm theoretical results.
\end{abstract}

\section{INTRODUCTION}

We consider the problem

$$
\begin{aligned}
& \frac{\partial u(t)}{\partial t}+A u(t)=f(t, u(t)), \quad t \in(0,1], \\
& u(0)=u_{0},
\end{aligned}
$$

where $u(t)$ is an unknown vector valued function with values in a Banach space $X$, $u_{0} \in X$ is a given vector, $f(t, u):\left(\mathbb{R}_{+} \times X\right) \rightarrow X$ is a given function (nonlinear operator) and $A$ is a linear densely defined closed operator with the domain $D(A)$ acting in $X$. The abstract setting (1.1) covers many applied problems such as nonlinear heat conduction or diffusion in porous media, the flow of electrons and holes in semiconductors, nerve axon equations, chemically reacting systems, equations of the population genetics theory, dynamics of nuclear reactors, Navier-Stokes equations of the viscous flow, etc. (see e.g. [26] and the references therein). This fact together with theoretical interest are important reasons to study efficient discrete approximations of problem (1.1).

Given a discretization parameter $N$ we are interesting in approximations possessing an exponential convergence rate with respect to $N \rightarrow \infty$ which for a given tolerance $\varepsilon$ provide algorithms of optimal or low complexity [14, 13]. Exponentially convergent algorithms were proposed recently for various linear problems.

Such algorithms for linear homogeneous parabolic problems of the type (1.1) were proposed probably first in 17, 19 and later in 11, 14, 20, 35, 40. These algorithms are based on a representation of the operator exponential $T(t)=e^{-A t}$

Received by the editor March 15, 2005 and, in revised form, June 30, 2006.

2000 Mathematics Subject Classification. Primary 65J15, 65M15; Secondary 34G20, 35K90.

Key words and phrases. Nonlinear evolution equation, exponentially convergent algorithms, Sinc-methods. 
by the improper Dunford-Cauchy integral along a path enveloping the spectrum of $A$ where a hyperbola containing the spectrum of $A$ or a parabola as the integration path were used. The methods from [20, 17, 11, 23, use Sinc-quadratures [32, 37. and possess an exponential convergence rate. An exponential convergence rate for all $t \geq 0$ was proven in [12, 41] under assumptions that the initial function $u_{0}$ belongs to the domain of $D\left(A^{\sigma}\right)$ for some $\sigma>1$, where the preliminary computation of $A^{\sigma} u_{0}$ is needed. Note that all these algorithms cannot be directly applied to inhomogeneous problems due to the inefficiency of computation of the operator exponential at $t=0$. In [20 a hyperbola as the integration path and a proper modification of the resolvent were used which allows one to get the uniform and numerically stable exponential convergence rate with respect to $t \geq 0$ without preliminary computation of $A^{\sigma} u_{0}$. An exponentially convergent algorithm for the case of an operator family $A(t)$ depending on the parameter $t$ was proposed in [16. This algorithm uses an exponentially convergent algorithm for the operator exponential generating by a constant operator. Moreover, these algorithms inherit two levels of parallelism (with respect to various time points and with respect to the treatment of the summands in the quadrature sum) which was perhaps first observed in the paper 34 and independently for the exponentially convergent algorithms in 17, 19, 11. A parallel method for the numerical solution of an integro-differential equation with positive memory was described in 28 .

In [14, 12, 13, 10] exponentially convergent algorithms based on the DunfordCauchy integral representation and on the Sinc-quadratures were proposed for parabolic and elliptic solution operators, for the Silvester operator equation, for the inverse of an elliptic operator and other operator-valued functions. Moreover, the paper [13] combines exponentially convergent algorithms with tensor product approximations in order to obtain algorithms of almost linear complexity (with respect to the discretization parameter in one dimension only) for high dimensional problems. The paper [6] deals with exponentially convergent algorithms for parabolic PDE's based on the Runge-Kutta methods. In [29, 30] exponentially convergent algorithms for inverting Laplace transforms were proposed and justified. The paper [4] deals with exponentially convergent algorithms for the first order differential equations with an operator coefficient possessing a variable domain. But the exponentially convergent methods for nonlinear parabolic problems in an abstract framework are missing in the literature.

In the present paper which represents a development of the technical report 22 we construct exponentially convergent approximations to the solution of nonlinear problem (1.1). To this end we use an equivalent Volterra integral equation including the operator exponential and represent the operator exponential by a Dunford-Cauchy integral along a hyperbola enveloping the spectrum of the operator coefficient. Then we approximate the integrals involved using the Chebyshev interpolation and an appropriate Sinc quadrature.

Problem (1.1) is equivalent to the nonlinear Volterra integral equation

$$
u(t)=u_{h}(t)+u_{n l}(t)
$$

where

$$
u_{h}(t)=T(t) u_{0}
$$


$T(t)=e^{-A t}$ is the operator exponential (the semi-group ) generated by $A$ and the nonlinear term is given by

$$
u_{n l}(t)=\int_{0}^{t} e^{-A(t-s)} f(s, u(s)) d s .
$$

We suppose that the solution $u(t)$ and the function $f(t, u(t))$ can be analytically extended (with respect to $t$ ) into a domain which we will describe below.

The aims of this paper are to propose a suitable abstract framework covering typical applied nonlinear problems relative to (1.1) and allowing exponentially convergent (for certain holomorphic right-hand sides), parallelizable approximations. In this sense the present paper extends the problem classes from the papers cited above for which it is possible to construct efficient algorithms with exponential accuracy.

The paper is organized as follows. In Section 2 using results from [20] we represent the operator exponential by the Dunford-Cauchy integral along a hyperbola enveloping the spectral angle of the operator $A$ which implies that the Volterra integral equation (1.2) contains two integrals: the first one over a variable but finite time interval and the second one over the real axis. The first integral is then approximated by the Gauss-Chebyshev quadrature and the second one by a Sinc quadrature rule. This procedure leads to a system of nonlinear algebraic equations which can be solved by an iteration method. Section 4 is devoted to the error analysis of this algorithm in the case of a small Lipschitz constant. Under some natural assumption on the spectral properties of the unbounded operator $A$ and on the nonlinear term $f(t, u)$ we show that our algorithm possesses a uniform with respect to $t$ exponential convergence rate. As an auxiliary result which we could not find in the literature on approximation theory we proved the following estimates for the classical Lebesque functions $\Lambda_{j}^{(1)}(\xi)=\sum_{k=1}^{N}\left|\int_{-1}^{\xi} \chi_{j}(\eta) L_{k, N-1}(\eta) d \eta\right| \leq \kappa_{j} \sqrt{\pi(\xi+1)}, \quad \Lambda_{j}^{(2)}(\xi)=$ $\sum_{k=1}^{N}\left|\int_{\xi}^{x_{j, N}} \chi_{j}(\eta) L_{k, N-1}(\eta) d \eta\right| \leq \kappa_{j} \sqrt{\pi\left(x_{j, N}-\xi\right)}, \xi \in\left(-1, x_{j, N}\right), j=1, \ldots, N$, where $L_{k, N-1}(\eta)$ are the Lagrange fundamental polynomials subject to the Chebyshev nodes $x_{j, N}$ and $\left|\chi_{j}(\eta)\right| \leq \kappa_{j} \forall \eta \in[-1,1], j=1, \ldots, N$, are some bounded functions.

Section 5 is concerned with a modification and with the analysis of the numerical algorithm from Section 4 for the case of an arbitrary Lipschitz constant.

In Section 6 we discuss the implementation of our algorithm. Several numerical and analytical examples are given throughout the text in order to make clear or to confirm the theoretical results.

\section{EXPONENTIALLY CONVERGENT APPROXIMATION TO THE OPERATOR EXPONENTIAL}

Let $A$ be a densely defined strongly positive operator in a Banach space $X$ with the domain $D(A)$, i.e. its spectrum $\Sigma(A)$ lies in the sector

$$
\Sigma=\left\{z=a_{0}+r e^{i \theta}: r \in[0, \infty),|\theta|<\varphi<\frac{\pi}{2}\right\}
$$

and on its boundary $\Gamma_{\Sigma}$, and outside of the sector the following estimate for the resolvent holds true:

$$
\left\|(z I-A)^{-1}\right\| \leq \frac{M}{1+|z|}
$$




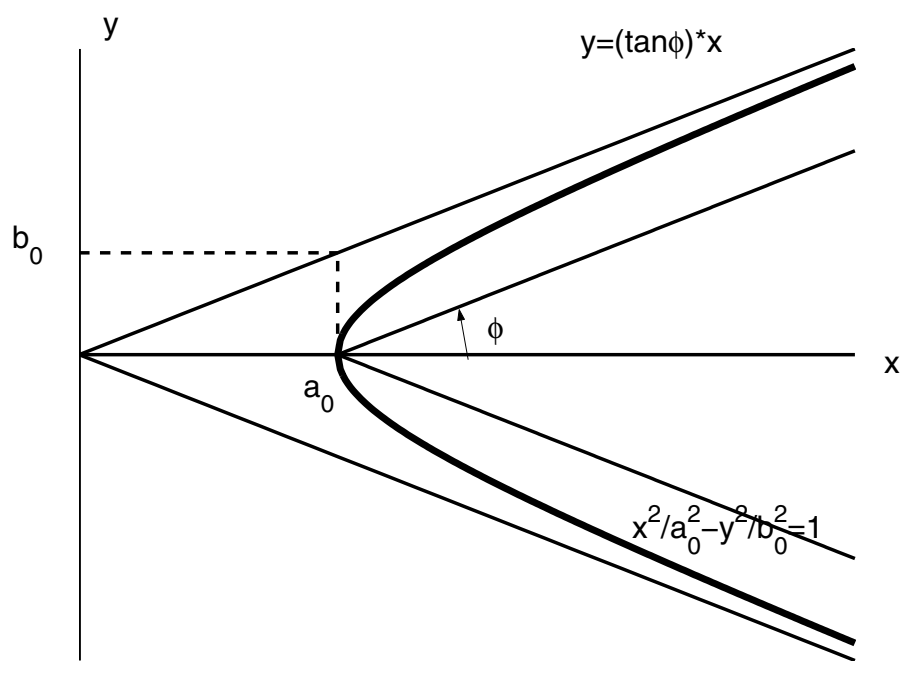

FIGURE 1. Spectral characteristics of the operator $A$.

with some positive constant $M$ (compare with [15, 27, 33, 36]). The angle $\varphi$ is called the spectral angle of the operator $A$. A practically important example of strongly positive operators represents a strongly elliptic partial differential operator [7, 15, 16. 33. where the parameters $a_{0}, \varphi$ of the sector $\Sigma$ are defined by its coefficients. We call the hyperbola

$$
\Gamma_{0}=\left\{z(\xi)=a_{0} \cosh \xi-i b_{0} \sinh \xi: \xi \in(-\infty, \infty), b_{0}=a_{0} \tan \varphi\right\}
$$

the spectral hyperbola, which pathes through the vertex $\left(a_{0}, 0\right)$ of the spectral angle and possesses asymptotes which are parallel to the rays of the spectral angle $\Sigma$. We choose the following hyperbola as an integration path [20]:

$$
\Gamma_{I}=\left\{z(\xi)=a_{I} \cosh \xi-i b_{I} \sinh \xi: \xi \in(-\infty, \infty)\right\},
$$

where

$$
\begin{aligned}
& a_{I}=a_{0} \cos \left(\frac{\pi}{4}-\frac{\varphi}{2}\right)-b_{0} \sin \left(\frac{\pi}{4}-\frac{\varphi}{2}\right) \\
& =\sqrt{a_{0}^{2}+b_{0}^{2}} \cos \left(\frac{\pi}{4}+\frac{\varphi}{2}\right)=a_{0} \frac{\cos \left(\frac{\pi}{4}+\frac{\varphi}{2}\right)}{\cos \varphi} \\
& b_{I}=a_{0} \sin \left(\frac{\pi}{4}-\frac{\varphi}{2}\right)+b_{0} \cos \left(\frac{\pi}{4}-\frac{\varphi}{2}\right) \\
& =\sqrt{a_{0}^{2}+b_{0}^{2}} \sin \left(\frac{\pi}{4}+\frac{\varphi}{2}\right)=a_{0} \frac{\sin \left(\frac{\pi}{4}+\frac{\varphi}{2}\right)}{\cos \varphi}
\end{aligned}
$$

Obviously this integration path envelops the spectral hyperbola but does not intersect it. Since the operator $A$ is strongly positive it holds on the integration path and outside of it:

$$
\begin{aligned}
& \left\|(z I-A)^{-1} w\right\| \leq \frac{M}{1+|z|}\|w\|, \\
& \left\|A(z I-A)^{-1} w\right\| \leq(1+M)\|w\| .
\end{aligned}
$$


Now, Theorem 4 from [27] yields

$$
\begin{aligned}
& \left\|A^{1-\alpha}(z I-A)^{-1} w\right\| \leq K\left\|A(z I-A)^{-1} w\right\|^{1-\alpha}\left\|(z I-A)^{-1} w\right\|^{\alpha}, \\
& \forall w \in X, \alpha \in[0,1],
\end{aligned}
$$

where the constant $K$ depends on $\alpha$ and $M$ only.

We consider the following representation of the operator exponential on an element $u_{0}$ :

$$
u_{h}(t)=\frac{1}{2 \pi i} \int_{\Gamma_{I}} e^{-z t}(z I-A)^{-1} u_{0} d z .
$$

One can also represent

$$
u_{h}(t)=\frac{1}{2 \pi i} \int_{\Gamma_{I}} e^{-z t}\left[(z I-A)^{-1}-\frac{1}{z} I\right] u_{0} d z
$$

instead of (2.8) (for $t>0$ the integral from the second summand is equal to zero due to the analyticity of the integrand inside of the integration path), and this integral represents the solution of the homogeneous problem (1.1) for $u_{0} \in D\left(A^{\alpha}\right), \alpha>0$. For the modified resolvent the following estimate holds true 20]:

$$
\begin{aligned}
& \left\|\left[(z I-A)^{-1}-\sum_{k=1}^{m+1} \frac{A^{k-1}}{z^{k}}\right] u_{0}\right\|=\left\|\frac{1}{z^{m+1}}(z I-A)^{-1} A^{m+1} u_{0}\right\| \\
& =\frac{1}{|z|^{m+1}}\left\|A^{1-\alpha}(z I-A)^{-1} A^{m+\alpha} u_{0}\right\| \leq \frac{1}{|z|^{m+1}} \frac{(1+M) K}{(1+|z|)^{\alpha}}\left\|A^{m+\alpha} u_{0}\right\|, \\
& \forall \alpha \in[0,1],
\end{aligned}
$$

provided that $u_{0} \in D\left(A^{m+\alpha}\right)$.

After parametrizing integral (2.9) by (2.4) we get

$$
u_{h}(t)=\frac{1}{2 \pi i} \int_{-\infty}^{\infty} \mathcal{F}(t, \xi) d \xi
$$

with

$$
\begin{aligned}
& \mathcal{F}(t, \xi)=F_{A}(t, \xi) u_{0}, \\
& F_{A}(t, \xi)=e^{-z(\xi) t}\left(a_{I} \sinh \xi-i b_{I} \cosh \xi\right)\left[(z(\xi) I-A)^{-1}-\frac{1}{z(\xi)} I\right] .
\end{aligned}
$$

It was shown in $[20$ that

$$
\|\mathcal{F}(t, \xi)\| \leq C(\varphi, \alpha) e^{-\alpha|\xi|}\left\|A^{\alpha} u_{0}\right\|, \xi \in \mathbb{R}, t \geq 0,
$$

with $C(\varphi, \alpha)=(1+M) K \tan \left(\frac{\pi}{4}+\frac{\varphi}{2}\right)\left(\frac{2 \cos \varphi}{a_{0} \cos \left(\frac{\pi}{4}+\frac{\varphi}{2}\right)}\right)^{\alpha}$. The change of $\xi \in \mathbb{R}$ to $w=\xi+i \nu \in \mathbb{C}$ implies that the integration hyperbola will be translated into the parametric set of hyperbolas

$$
\begin{aligned}
& \Gamma(\nu)=\left\{z(w)=a_{I} \cosh (\xi+i \nu)-i b_{I} \sinh (\xi+i \nu): \xi \in(-\infty, \infty)\right\} \\
& =\{z(w)=a(\nu) \cosh \xi-i b(\nu) \sinh \xi: \xi \in(-\infty, \infty)\}
\end{aligned}
$$


so that $\Gamma(0)=\Gamma_{I}$, where 20 ]

$$
\begin{aligned}
a(\nu) & =a_{I} \cos \nu+b_{I} \sin \nu=\frac{a_{0} \sin (\nu+\pi / 4-\varphi / 2)}{\cos \varphi} \\
& =\frac{a_{0} \cos (\pi / 4+\varphi / 2-\nu)}{\cos \varphi}, \\
b(\nu) & =b_{I} \cos \nu-a_{I} \sin \nu=\frac{a_{0} \sin (\pi / 4+\varphi / 2-\nu)}{\cos \varphi} .
\end{aligned}
$$

The vector valued function $\mathcal{F}(t, w)$ is analytic with respect to $w=\xi+i \nu$ in the strip

$$
D_{d_{1}}=\left\{w=\xi+i \nu: \xi \in(-\infty, \infty),|\nu|<d_{1} / 2\right\},
$$

where $d_{1}=\pi / 2-\varphi$ for all $t \geq 0$. Moreover, for an arbitrarily small positive $\delta$ in the strip $D_{d}$ with $d=d_{1}-\delta$ there holds

$$
\begin{aligned}
& \|\mathcal{F}(t, w)\| \\
& \leq(1+M) K \tan \left(\frac{\pi}{4}+\frac{\varphi}{2}-\nu\right)\left(\frac{2 \cos \varphi}{a_{0} \cos (\pi / 4+\varphi / 2-\nu)}\right)^{\alpha} e^{-\alpha|\xi|}\left\|A^{\alpha} u_{0}\right\|,
\end{aligned}
$$

$\forall w \in D_{d}$.

It was also shown that

$$
\begin{aligned}
& \left|z^{\prime}(w) / z(w)\right| \leq b(\nu) / a(\nu)=\tan (\pi / 4+\varphi / 2-\nu), \\
& \left|z^{\prime}(w)\right| /|z(w)|^{1+\alpha} \leq \tan \left(\frac{\pi}{4}+\frac{\varphi}{2}-\nu\right)\left(\frac{2 \cos \varphi}{a_{0} \cos \left(\frac{\pi}{4}+\frac{\varphi}{2}-\nu\right)}\right)^{\alpha},
\end{aligned}
$$

which implies

$$
\|\mathcal{F}(t, \cdot)\|_{H^{1}\left(D_{d}\right)} \leq C(\varphi, \alpha, \delta)\left\|A^{\alpha} u_{0}\right\|
$$

where

$$
\begin{aligned}
& C(\varphi, \alpha, \delta)=\frac{2}{\alpha}\left[C_{+}(\varphi, \alpha, \delta)+C_{-}(\varphi, \alpha, \delta)\right] \\
& C_{ \pm}(\varphi, \alpha, \delta)=(1+M) K(\cos \varphi)^{\alpha} \tan \left(\frac{\pi}{4}+\frac{\varphi}{2} \pm \frac{d}{2}\right)\left(\frac{2}{a_{0} \cos \left(\frac{\pi}{4}+\frac{\varphi}{2} \pm \frac{d}{2}\right)}\right)^{\alpha}
\end{aligned}
$$

and the constant $C(\varphi, \alpha, \delta)$ tends to $\infty$ if $\alpha \rightarrow 0$ or $\delta \rightarrow 0, \varphi \rightarrow \pi / 2$.

We approximate integral (2.11) by the following Sinc-quadrature:

$$
u_{N}(t)=e_{N}^{-A t} u_{0}=\frac{h}{2 \pi i} \sum_{k=-N}^{N} \mathcal{F}(t, z(k h)),
$$

where

$$
e_{N}^{-A t}=\frac{h}{2 \pi i} \sum_{k=-N}^{N} F_{A}(t, z(k h))
$$


is an approximation to the operator exponential. For the error we have the estimate (see [20])

$$
\begin{aligned}
& \left\|\eta_{N}(\mathcal{F}, h)\right\|=\left\|u(t)-u_{N}(t)\right\| \\
& \leq \frac{c\left\|A^{\alpha} u_{0}\right\|}{\alpha}\left\{\frac{e^{-\pi d / h}}{\sinh (\pi d / h)}+\exp \left[-a_{I} t \cosh ((N+1) h)-\alpha(N+1) h\right]\right\},
\end{aligned}
$$

where the constant $c$ does not depend on $h, N, t$.

Moreover, it is easy to see that

$$
\begin{aligned}
& \left\|A^{\beta} \eta_{N}(\mathcal{F}, h)\right\|=\left\|A^{\beta}\left(u(t)-u_{N}(t)\right)\right\| \\
& \leq \frac{c\left\|A^{\alpha+\beta} u_{0}\right\|}{\alpha}\left\{\frac{e^{-\pi d / h}}{\sinh (\pi d / h)}+\exp \left[-a_{I} t \cosh ((N+1) h)-\alpha(N+1) h\right]\right\} \\
& \quad \forall \beta \geq 0, \quad \forall \alpha>0 .
\end{aligned}
$$

Since we also apply this quadrature later for $t \rightarrow 0$, it is important that it converges exponentially for all $t \geq 0$. Equalizing both exponentials for $t=0$ by

$$
\frac{2 \pi d}{h}=\alpha(N+1) h
$$

we get for the step size

$$
h=\sqrt{\frac{2 \pi d}{\alpha(N+1)}}
$$

the following error estimates:

$$
\left\|\eta_{N}(\mathcal{F}, h)\right\| \leq \frac{c}{\alpha} \exp \left(-\sqrt{\frac{\pi d \alpha}{2}(N+1)}\right)\left\|A^{\alpha} u_{0}\right\|
$$

and

$$
\left\|A^{\beta} \eta_{N}(\mathcal{F}, h)\right\| \leq \frac{c}{\alpha} \exp \left(-\sqrt{\frac{\pi d \alpha}{2}(N+1)}\right)\left\|A^{\alpha+\beta} u_{0}\right\| \quad \forall \beta \geq 0, \quad \forall \alpha>0
$$

with a constant $c$ independent of $t, N$.

The first summand in the exponent of $e^{\left[-a_{I} t \cosh ((N+1) h)-\alpha(N+1) h\right]}$ contributes mainly to the error order. Setting $h=c_{1} \ln N / N$ with some positive constant $c_{1}$ in (2.23) we remain asymptotically for a fixed $t>0$ with an error

$$
\left\|\eta_{N}(\mathcal{F}, h)\right\| \leq c\left[e^{-\pi d N /\left(c_{1} \ln N\right)}+e^{-c_{1} a_{I} t N / 2-c_{1} \alpha \ln N}\right]\left\|A^{\alpha} u_{0}\right\|,
$$

where $c$ is a positive constant. Thus, we have proven the following result.

Theorem 2.1. Let $A$ be a densely defined, closed, strongly positive linear operator and $u_{0} \in D\left(A^{\alpha}\right), \alpha \in(0,1)$. Then Sinc-quadrature (2.21) represents an approximate solution of the homogeneous initial value problem (1.1) (i.e. $\left.u(t)=e^{-A t} u_{0}\right)$ and possesses a uniform with respect to $t \geq 0$ exponential convergence rate with estimate (2.23) which is of the order $\mathcal{O}\left(e^{-c \sqrt{N}}\right)$ uniformly in $t \geq 0$ provided that $h=\mathcal{O}(1 / \sqrt{N})$ and of the order

$$
\mathcal{O}\left(\max \left\{e^{-\pi d N /\left(c_{1} \ln N\right)}, e^{-c_{1} a_{I} t N / 2-c_{1} \alpha \ln N}\right\}\right)
$$

for each fixed $t \geq 0$ provided that $h=c_{1} \ln N / N$. 


\section{A discretization scheme of Chebyshev type}

Changing in (1.2) the variables by

$$
t=\frac{x+1}{2}
$$

we transform problem (1.2) to the following problem on the interval $[-1,1]$ :

$$
u\left(\frac{x+1}{2}\right)=g_{h}(x)+g_{n l}(x, u)
$$

with

$$
\begin{aligned}
& g_{h}(x)=e^{-A \frac{x+1}{2}} u_{0}, \\
& g_{n l}(x, u)=\frac{1}{2} \int_{-1}^{x} e^{-A \frac{x-\xi}{2}} f\left(\frac{\xi+1}{2}, u\left(\frac{\xi+1}{2}\right)\right) d \xi
\end{aligned}
$$

Using the representation of the operator exponential by the Dunford-Cauchy integral along the integration path $\Gamma_{I}$ defined above in (2.4), (2.5) and enveloping the spectral curve $\Gamma_{0}$ we obtain

$$
\begin{aligned}
& g_{h}(x)=e^{-A \frac{x+1}{2}} u_{0}=\frac{1}{2 \pi i} \int_{\Gamma_{I}} e^{-z \frac{x+1}{2}}\left[(z I-A)^{-1}-\frac{1}{z} I\right] u_{0} d z, \\
& g_{n l}(x, u)=\frac{1}{2} \int_{-1}^{x} e^{-A \frac{x-\eta}{2}} f\left(\frac{\eta+1}{2}, u\left(\frac{\eta+1}{2}\right)\right) d \eta \\
& =\frac{1}{4 \pi i} \int_{-1}^{x} \int_{\Gamma_{I}} e^{-z \frac{x-\eta}{2}}\left[(z I-A)^{-1}-\frac{1}{z} I\right] f\left(\frac{\eta+1}{2}, u\left(\frac{\eta+1}{2}\right)\right) d z d \eta
\end{aligned}
$$

(note, that P.V. $\int_{\Gamma_{I}} z^{-1} d z=0$, but this term in the resolvent provides the numerical stability of the algorithm below when $t \rightarrow 0$; see 20] for details). After parametrizing the first integral in (3.4) by (2.4) we have

$$
g_{h}(x)=\frac{1}{2 \pi i} \int_{-\infty}^{\infty} \mathcal{F}_{h}(x, \xi) d \xi
$$

with

$$
\mathcal{F}_{h}(x, \xi)=F_{A}((x+1) / 2, \xi) u_{0}
$$

(in the case $A=0$ we define $F_{A}(t, \xi)=0$ ).

We approximate integral (3.5) by the following Sinc-quadrature (see (2.21), (2.26), (2.27)):

$$
g_{h, N_{1}}(x)=\frac{h}{2 \pi i} \sum_{k=-N_{1}}^{N_{1}} \mathcal{F}_{h}(x, k h), \quad h=\sqrt{\frac{2 \pi d}{\alpha\left(N_{1}+1\right)}},
$$

with the error

$$
\left\|\eta_{N_{1}}\left(\mathcal{F}_{h}, h\right)\right\|=\left\|\mathcal{E}((x+1) / 2) u_{0}\right\| \leq \frac{c}{\alpha} \exp \left(-\sqrt{\frac{\pi d \alpha}{2}\left(N_{1}+1\right)}\right)\left\|A^{\alpha} u_{0}\right\|,
$$

where

$$
\mathcal{E}((x-\eta) / 2)=\frac{1}{2 \pi i} \int_{-\infty}^{\infty} F_{A}((x-\eta) / 2, \xi) d \xi-\frac{1}{2 \pi i} \sum_{k=-N_{1}}^{N_{1}} F_{A}((x-\eta) / 2, k h)
$$


and the constant $c$ independent of $x, N_{1}$. Analogously we transform the second integral in (3.4) to

$$
\begin{aligned}
& g_{n l}(x, u)=\frac{1}{4 \pi i} \int_{-1}^{x} \int_{\Gamma_{I}} e^{-z \frac{x-\eta}{2}}\left[(z I-A)^{-1}-\frac{1}{z} I\right] f\left(\frac{\eta+1}{2}, u\left(\frac{\eta+1}{2}\right)\right) d z d \eta \\
& =\frac{1}{4 \pi i} \int_{-1}^{x} \int_{-\infty}^{\infty} \mathcal{F}_{n l}(x, \xi, \eta) d \xi d \eta,
\end{aligned}
$$

where

$$
\mathcal{F}_{n l}(x, \xi, \eta)=F_{A}((x-\eta) / 2, \xi) f\left(\frac{\eta+1}{2}, u\left(\frac{\eta+1}{2}\right)\right) .
$$

Replacing the infinite integral by quadrature rule (3.7) we arrive at the approximation

$$
g_{n l, N_{1}}(x, u)=\frac{h}{4 \pi i} \int_{-1}^{x} \sum_{k=-N_{1}}^{N_{1}} \mathcal{F}_{n l}(x, k h, \eta) d \eta .
$$

In order to approximate the nonlinear operator $g_{n l, N_{1}}(x, u)$ we choose the mesh $\omega_{N}=\left\{x_{k, N}=\cos \frac{(2 k-1) \pi}{2 N}, k=1, \ldots, N\right\}$ on $[-1,1]$, where $x_{k, N}$ are zeros of Chebyshev orthogonal polynomial of first kind $T_{N}(x)=\cos (N \arccos x)$. For the stepsizes $\tau_{k, N}=x_{k, N}-x_{k-1, N}$ it is well known that (see [38, Ch.6, Th.6.11.12, 39], p. 123)

$$
\begin{gathered}
\tau_{k, N}=x_{k+1, N}-x_{k, N}<\frac{\pi}{N}, k=1, \ldots, N, \\
\tau_{\max }=\max _{1 \leq k \leq N} \tau_{k, N}<\frac{\pi}{N} .
\end{gathered}
$$

Let

$$
P_{N-1}(x ; f(\cdot, u))=\sum_{p=1}^{N} f\left(\left(x_{p, N}+1\right) / 2, u\left(\left(x_{p, N}+1\right) / 2\right)\right) L_{p, N-1}(x)
$$

be the interpolation polynomial for the function $f(x, u(x))$ on the mesh $\omega_{N}$, i.e. $P_{N-1}\left(x_{k, N} ; f(\cdot, u)\right)=f\left(\left(x_{k, N}+1\right) / 2, u\left(\left(x_{k, N}+1\right) / 2\right)\right), k=1,2, \ldots, N$, where $L_{p, N-1}$ $=\frac{T_{N}(x)}{T_{N}^{\prime}\left(x_{p, N}\right)\left(x-x_{p, N}\right)}, p=1, \ldots, N$, are the Lagrange fundamental polynomials. Given a vector $y=\left(y_{1}, \ldots, y_{N}\right), y_{i} \in X$, let

$$
P_{N-1}(x ; f(\cdot, y))=\sum_{p=1}^{N} f\left(\left(x_{p, N}+1\right) / 2, y_{p}\right) L_{p, N-1}(x)
$$

be the polynomial which interpolates $f(x, y)$, i.e.

$$
P_{N-1}\left(x_{k, N} ; f(\cdot, y)\right)=f\left(\left(x_{k, N}+1\right) / 2, y_{k}\right), k=1,2, \ldots, N .
$$

Substituting $P_{N-1}(t ; f(\cdot, y))$ instead of $f(t, u)$ into (3.11), (3.12) we get the approximation

$$
g_{n l, N, N_{1}}(x, y)=\frac{h}{4 \pi i} \int_{-1}^{x} \sum_{k=-N_{1}}^{N_{1}} F_{A}((x-\eta) / 2, k h) P_{N-1}(\eta ; f(\cdot, y)) d \eta .
$$

Substituting approximations (3.7) and (3.16) into (3.2) and collocating the resulting equation on the grid $\omega_{N}$ we arrive at the following Algorithm A1 for solving problem (3.2): find $y=\left(y_{1}, \ldots, y_{N}\right), y_{i} \in X$, such that

$$
y_{j}=g_{h, N_{1}}\left(x_{j, N}\right)+g_{n l, N, N_{1}}\left(x_{j, N}, y\right), j=1, \ldots, N,
$$


or

$$
\begin{aligned}
& y_{j}=\frac{h}{2 \pi i} \sum_{k=-N_{1}}^{N_{1}} \mathcal{F}_{h}\left(x_{j, N}, k h\right) \\
& +\frac{h}{4 \pi i} \sum_{k=-N_{1}}^{N_{1}} \int_{-1}^{x_{j, N}} F_{A}\left(\left(x_{j, N}-\eta\right) / 2, k h\right) P_{N-1}(\eta ; f(\cdot, y)) d \eta, \\
& j=1, \ldots, N .
\end{aligned}
$$

Equations (3.17) or (3.18) define a nonlinear operator $\mathcal{A}$ so that

$$
y=\mathcal{A}(y)+\phi,
$$

where

$$
\begin{aligned}
& y=\left(y_{1}, y_{2}, \ldots, y_{N}\right), \quad y_{i} \in X, \\
& {[\mathcal{A}(y)]_{j}=\frac{h}{4 \pi i} \sum_{k=-N_{1}}^{N_{1}} \int_{-1}^{x_{j, N}} F_{A}\left(\left(x_{j, N}-\eta\right) / 2, k h\right) P_{N-1}(\eta ; f(\cdot, y)) d \eta,} \\
& (\phi)_{j}=\frac{h}{2 \pi i} \sum_{k=-N_{1}}^{N_{1}} \mathcal{F}_{h}\left(x_{j, N}, k h\right)=\frac{h}{2 \pi i} \sum_{k=-N_{1}}^{N_{1}} F_{A}\left(\left(x_{j, N}+1\right) / 2, k h\right) u_{0}, \\
& j=1, \ldots, N .
\end{aligned}
$$

This is a system of nonlinear equations which can be solved by an iteration method. Since the integrands in

$$
I_{j, k}=\int_{-1}^{x_{j, N}} F_{A}\left(\left(x_{j, N}-\eta\right) / 2, k h\right) P_{N-1}(\eta ; f(\cdot, y)) d \eta, j=1, \ldots, N, k=-N_{1}, \ldots, N_{1},
$$

are products of the exponential function and polynomials, these integrals can be calculated analytically, for example, by computer algebra tools.

Given the vector $y=\left(y_{1}, \ldots, y_{N}\right)$ the interpolation polynomial $\tilde{u}(x)=P_{N-1}(x ; y)$ represents an approximation for $u((x+1) / 2)=u(t)$, i.e. $u((x+1) / 2)=u(t) \approx$ $P_{N-1}(x ; y)$.

\section{The ERROR ANALYSIS FOR A SMALl LiPSCHITZ CONSTANT}

In this section we investigate the error of algorithm (3.18). The projection of the exact equation (3.2) onto the grid $\omega_{N}$ provides

$$
\begin{aligned}
& u\left(t_{j}\right)=e^{-A t_{j}} u_{0}+\frac{1}{2} \int_{-1}^{x_{j, N}} e^{-A\left(x_{j, N}-\xi\right) / 2} f\left(\frac{1+\xi}{2}, u\left(\frac{1+\xi}{2}\right)\right) d \xi, \\
& t_{j}=\frac{1+x_{j, N}}{2}, \quad x_{j, N}=\cos \frac{(2 j-1) \pi}{2 N}, \quad j=1, \ldots, N .
\end{aligned}
$$

Using equations (3.18) we represent the error of the algorithm in the form

$$
\begin{aligned}
& Z_{j}=u\left(t_{j}\right)-y_{j} \\
& =\psi_{j}^{(0)}+\psi_{j}^{(1)}+\psi_{j}^{(2)}+\psi_{j}^{(3)}, \quad j=1, \ldots, N,
\end{aligned}
$$


where

$(4.3)$

$$
\begin{aligned}
& \psi_{j}^{(0)}=\eta_{N}\left(\mathcal{F}_{h}, h\right) \\
& =\left\{e^{-A t_{j}}-\frac{h}{2 \pi i} \sum_{k=-N_{1}}^{N_{1}} z^{\prime}(k h) e^{-t_{j} z(k h)}\left[(z(k h) I-A)^{-1}-\frac{1}{z(k h)} I\right] u_{0}\right. \\
& \psi_{j}^{(1)}=\frac{1}{2} \int_{-1}^{x_{j, N}} f\left(\frac{1+\eta}{2}, u\left(\frac{1+\eta}{2}\right)\right)\left\{e^{-A \frac{\left(x_{j, N}-\eta\right)}{2}}{ }^{N_{1}} z^{\prime}(k h) e^{-z(k h) \frac{\left(x_{j, N}-\eta\right)}{2}}\left[(z(k h) I-A)^{-1}-\frac{1}{z(k h)} I\right] d \eta,\right. \\
& -\frac{h}{2 \pi i} \sum_{k=-N_{1}}{ }^{(2)}=\frac{h}{2 \pi i} \sum_{k=-N_{1}}^{N_{1}} z^{\prime}(k h) \frac{1}{2} \int_{-1}^{x_{j, N}} e^{-z(k h)\left(x_{j, N}-\eta\right) / 2}\left[(z(k h) I-A)^{-1}-\frac{1}{z(k h)} I\right] \\
& \left.\times\left[\frac{1+\eta}{2}, u\left(\frac{1+\eta}{2}\right)\right)-\sum_{l=1}^{N} f\left(t_{l}, u\left(t_{l}\right)\right) L_{l, N-1}(\eta)\right] d \eta, \\
& \psi_{j}^{(3)}=\frac{h}{2 \pi i} \sum_{k=-N_{1}}^{N_{1}} z^{\prime}(k h) \frac{1}{2} \int_{-1}^{x_{j, N}} e^{-z(k h)\left(x_{j, N}-\eta\right) / 2}\left[(z(k h) I-A)^{-1}-\frac{1}{z(k h)} I\right] \\
& \times\left[\sum_{l=1}^{N}\left[f\left(t_{l}, u\left(t_{l}\right)\right)-f\left(t_{l}, y_{l}\right)\right] L_{l, N-1}(\eta)\right] d \eta,
\end{aligned}
$$

Using notations (3.6), (3.9) we can write down

$$
\begin{aligned}
& \psi_{j}^{(0)}=\mathcal{E}\left(\left(x_{j, N}+1\right) / 2\right) u_{0} \\
& \psi_{j}^{(1)}=\frac{1}{2} \int_{-1}^{x_{j, N}} \mathcal{E}\left(\left(x_{j, N}-\eta\right) / 2\right) f\left(\frac{1+\eta}{2}, u\left(\frac{1+\eta}{2}\right)\right) d \eta \\
& \psi_{j}^{(2)}=\frac{1}{2} \int_{-1}^{x_{j, N}} \frac{h}{2 \pi i} \sum_{k=-N_{1}}^{N_{1}} F_{A}\left(\left(x_{j, N}-\eta\right) / 2, k h\right) \\
& \times\left[f\left(\frac{1+\eta}{2}, u\left(\frac{1+\eta}{2}\right)\right)-P_{N-1}(\eta ; f(\cdot, u(\cdot)))\right] d \eta \\
& \psi_{j}^{(3)}=\frac{1}{2} \int_{-1}^{x_{j, N}} \frac{h}{2 \pi i} \sum_{k=-N_{1}}^{N_{1}} F_{A}\left(\left(x_{j, N}-\eta\right) / 2, k h\right) \\
& \times\left[P_{N-1}(\eta ; f(\cdot, u(\cdot))-f(\cdot, y(\cdot)))\right] d \eta \\
& =[\mathcal{A}(u)]_{j}-[\mathcal{A}(y)]_{j}
\end{aligned}
$$

where $u=\left(u\left(t_{1}\right), \ldots, u\left(t_{N}\right)\right), y=\left(y_{1}, \ldots, y_{N}\right)$ and $\psi_{j}=\psi_{j}^{(0)}+\psi_{j}^{(1)}+\psi_{j}^{(2)}$ is the truncation error.

For the first summand we have estimate (3.8):

$$
\left\|\psi_{j}^{(0)}\right\| \leq \frac{c}{\alpha} \exp \left(-\sqrt{\frac{\pi d \alpha}{2}\left(N_{1}+1\right)}\right)\left\|A^{\alpha} u_{0}\right\|
$$


and obviously the estimate

$$
\left\|A^{\alpha} \psi_{j}^{(0)}\right\| \leq \frac{c}{\beta-\alpha} \exp \left(-\sqrt{\frac{\pi d \alpha}{2}\left(N_{1}+1\right)}\right)\left\|A^{\beta} u_{0}\right\| \quad \forall \beta>\alpha>0 .
$$

In order to estimate $\psi_{j}^{(1)}$ we assume that

(i)

$$
f(t, u(t)) \in D\left(A^{\alpha}\right) \forall t \in[0,1] \text { and } \int_{0}^{1}\left\|A^{\alpha} f(t, u(t))\right\| d t<\infty .
$$

Using this assumption we obtain analogously to (4.5), (4.6)

$$
\left\|\psi_{j}^{(1)}\right\| \leq \frac{c}{\alpha} \exp \left(-\sqrt{\frac{\pi d \alpha}{2}\left(N_{1}+1\right)}\right) \int_{0}^{1}\left\|A^{\alpha} f(t, u(t))\right\| d t
$$

and

$$
\left\|A^{\alpha} \psi_{j}^{(1)}\right\| \leq \frac{c}{\beta-\alpha} \exp \left(-\sqrt{\frac{\pi d \alpha}{2}\left(N_{1}+1\right)}\right) \int_{0}^{1}\left\|A^{\beta} f(t, u(t))\right\| d t \quad \forall \beta>\alpha>0 .
$$

In order to estimate $\psi_{j}^{(2)}$ we assume in addition to (4.7) that

(ii) the vector valued function $A^{\alpha} f\left(\frac{1+\xi}{2}, u\left(\frac{1+\xi}{2}\right)\right)$ of $\xi$ can be analytically extended from the interval $B=[-1,1]$ into the domain $\mathcal{D}_{\rho}$ enveloped by the so called Bernstein's regularity ellipse $\mathcal{E}_{\rho}=\mathcal{E}_{\rho}(B)$ (with the foci at $z= \pm 1$ and the sum of semi-axes equal to $\rho>1$ ):

$$
\begin{gathered}
\mathcal{E}_{\rho}=\left\{z \in \mathbb{C}: z=\frac{1}{2}\left(\rho e^{i \varphi}+\frac{1}{\rho} e^{-i \phi}\right)\right\} \\
=\left\{(x, y): \frac{x^{2}}{a^{2}}+\frac{y^{2}}{b^{2}}=1, a=\frac{1}{2}\left(\rho+\frac{1}{\rho}\right), b=\frac{1}{2}\left(\rho-\frac{1}{\rho}\right)\right\} .
\end{gathered}
$$

Using (2.10) with $m=0$, the first inequality (2.18) with $\nu=0$ and the fact that the Lebesque constant for the Chebyshev interpolation process is bounded by $c \ln N$ we obtain

$$
\left\|\psi_{j}^{(2)}\right\| \leq c \cdot S_{N_{1}} \cdot \ln N \cdot E_{N}\left(A^{\alpha} f(\cdot, u(\cdot))\right)
$$

where $S_{N_{1}}=\sum_{k=-N_{1}}^{N_{1}} h\left|z^{\prime}(k h)\right| /|z(k h)|^{1+\alpha}, c$ is a constant independent of $N, N_{1}, \eta$ and $E_{N}\left(A^{\alpha} f(\cdot, u(\cdot))\right)$ is the value of the best approximation of $A^{\alpha} f(t, u(t))$ by polynomials of degree not greater than $N-1$ in the maximum norm with respect to $t$. Using the estimate

$$
\begin{aligned}
& |z(k h)|=\sqrt{a_{I}^{2} \cosh ^{2}(k h)+b_{I}^{2} \sinh ^{2}(k h)} \\
& \geq a_{I} \cosh (k h) \geq a_{I} e^{|k h|} / 2
\end{aligned}
$$

the last sum can be estimated by

$$
\left|S_{N_{1}}\right| \leq \frac{c}{\sqrt{N_{1}}} \sum_{k=-N_{1}}^{N_{1}} e^{-\alpha\left|k / \sqrt{N_{1}}\right|} \leq c \int_{-\sqrt{N_{1}}}^{\sqrt{N_{1}}} e^{-\alpha t} d t \leq c / \alpha
$$


Due to assumption (ii) we have for the value of the best polynomial approximation [5, 20]

$$
E_{N}\left(A^{\alpha} f(\cdot, u(\cdot))\right) \leq \frac{\rho^{-N}}{1-\rho} \sup _{z \in D_{\rho}}\left\|A^{\alpha} f(z, u(z))\right\|
$$

which together with (4.10) and (4.12) yields

$$
\left\|\psi_{j}^{(2)}\right\| \leq \frac{c}{\alpha} \ln N \rho^{-N} \sup _{z \in D_{\rho}}\left\|A^{\alpha} f(z, u(z))\right\|
$$

and

$$
\left\|A^{\alpha} \psi_{j}^{(2)}\right\| \leq \frac{c}{\beta-\alpha} \ln N \rho^{-N} \sup _{z \in D_{\rho}}\left\|A^{\beta} f(z, u(z))\right\| \quad \forall \beta>\alpha>0 .
$$

Before we go over to the estimating of $\psi_{j}^{(3)}$ let us introduce the functions

$$
\Lambda_{j}^{(1)}(\xi)=\sum_{k=1}^{N}\left|\int_{-1}^{\xi} \chi_{j}(\eta) L_{k, N-1}(\eta) d \eta\right|, j=1, \ldots, N,
$$

and

$$
\Lambda_{j}^{(2)}(\xi)=\sum_{k=1}^{N}\left|\int_{\xi}^{x_{j, N}} \chi_{j}(\eta) L_{k, N-1}(\eta) d \eta\right|, j=1, \ldots, N
$$

with some bounded functions $\chi_{j}(\eta)$ :

$$
\left|\chi_{j}(\eta)\right| \leq \kappa_{j} \forall \eta \in[-1,1], j=1, \ldots, N,
$$

and prove the following auxiliary assertion.

Lemma 4.1. There holds

$$
\begin{aligned}
& \Lambda_{j}^{(1)}(\xi) \leq \kappa_{j} \sqrt{\pi(\xi+1)}, \\
& \Lambda_{j}^{(2)}(\xi) \leq \kappa_{j} \sqrt{\pi\left(x_{j, N}-\xi\right)}, \xi \in\left(-1, x_{j, N}\right), j=1, \ldots, N .
\end{aligned}
$$

Proof. Let

$$
\begin{aligned}
& \epsilon_{k, j}^{(1)}=\operatorname{sign}\left\{\int_{-1}^{\xi} \chi_{j}(\eta) L_{k, N-1}(\eta) d \eta\right\}, \\
& \epsilon_{k, j}^{(2)}=\operatorname{sign}\left\{\int_{\xi}^{x_{j, N}} \chi_{j}(\eta) L_{k, N-1}(\eta) d \eta\right\} .
\end{aligned}
$$

Then taking into account that all coefficients of the Gauß quadrature relating to the Chebyshev orthogonal polynomials of the first kind are equal to $\pi / N$ and the Lagrange fundamental polynomials $L_{k, N}(\eta)$ are orthogonal 38, with the weight 
$1 / \sqrt{1-\eta^{2}}$ we obtain

$$
\begin{aligned}
& \Lambda_{j}^{(1)}(\xi)=\int_{-1}^{\xi} \chi_{j}(\eta) \sum_{k=1}^{N} \epsilon_{k, j}^{(1)} L_{k, N-1}(\eta) d \eta \\
& \leq \sqrt{\xi+1}\left\{\int_{-1}^{\xi} \chi_{j}^{2}(\eta)\left[\sum_{k=1}^{N} \epsilon_{k, j}^{(1)} L_{k, N-1}(\eta)\right]^{2} d \eta\right\}^{1 / 2} \\
& \leq \kappa_{j} \sqrt{\xi+1}\left\{\int_{-1}^{\xi}\left[\sum_{k=1}^{N} \epsilon_{k, j}^{(1)} L_{k, N-1}(\eta)\right]^{2} / \sqrt{1-\eta^{2}} d \eta\right\}^{1 / 2} \\
& \leq \kappa_{j} \sqrt{\xi+1}\left\{\sum_{k, p=1}^{N} \epsilon_{k, j}^{(1)} \epsilon_{p, j}^{(1)} \int_{-1}^{1} L_{k, N-1}(\eta) L_{p, N-1}(\eta) / \sqrt{1-\eta^{2}} d \eta\right\}^{1 / 2} \\
& =\kappa_{j} \sqrt{\xi+1}\left\{\sum_{k=1}^{N}\left(\epsilon_{k, j}^{(1)}\right)^{2} \int_{-1}^{1} L_{k, N-1}^{2}(\eta) / \sqrt{1-\eta^{2}} d \eta\right\}^{1 / 2} \\
& =\kappa_{j} \sqrt{\pi / N} \sqrt{\xi+1}\left\{\sum_{k=1}^{N} 1\right\}^{1 / 2} \\
& =\kappa_{j} \sqrt{\pi(1+\xi)} .
\end{aligned}
$$

Analogously we obtain

$$
\begin{aligned}
& \Lambda_{j}^{(2)}(\xi)=\int_{\xi}^{x_{j, N}} \chi_{j}(\eta) \sum_{k=1}^{N} \epsilon_{k, j}^{(2)} L_{k, N-1}(\eta) d \eta \\
& \leq \sqrt{x_{j, N}-\xi}\left\{\int_{\xi}^{x_{j, N}} \chi_{j}^{2}(\eta)\left[\sum_{k=1}^{N} \epsilon_{k, j}^{(2)} L_{k, N-1}(\eta)\right]^{2} d \eta\right\}^{1 / 2} \\
& \leq \kappa_{j} \sqrt{x_{j, N}-\xi}\left\{\int_{\xi}^{x_{j, N}}\left[\sum_{k=1}^{N} \epsilon_{k, j}^{(2)} L_{k, N-1}(\eta)\right]^{2} / \sqrt{1-\eta^{2}} d \eta\right\}^{1 / 2} \\
& \leq \kappa_{j} \sqrt{x_{j, N}-\xi}\left\{\sum_{k, p=1}^{N} \epsilon_{k, j}^{(2)} \epsilon_{p, j}^{(2)} \int_{-1}^{1} L_{k, N-1}(\eta) L_{p, N-1}(\eta) / \sqrt{1-\eta^{2}} d \eta\right\}^{1 / 2} \\
& =\kappa_{j} \sqrt{\pi\left(x_{j, N}-\xi\right)}=\sqrt{2 \pi} \kappa_{j} \cos \frac{(2 j-1) \pi}{4 N} .
\end{aligned}
$$

The proof is complete.

Corollary 4.2. We define the numbers

$$
\Lambda_{j}^{(1)}=\Lambda_{j}^{(1)}\left(x_{j, N}\right)=\sum_{k=1}^{N}\left|\int_{-1}^{x_{j, N}} \chi_{j}(\eta) L_{k, N-1}(\eta) d \eta\right|, j=1, \ldots, N .
$$


Then using Lemma 4.1 we derive

$$
\Lambda_{j}^{(1)} \leq \kappa_{j} \sqrt{\pi\left(1+\cos \frac{(2 j-1) \pi}{2 N}\right)}=\sqrt{2 \pi} \kappa_{j} \cos \frac{(2 j-1) \pi}{4 N}, j=1, \ldots, N .
$$

Conjecture. Setting $\chi(\eta)=1$, we obtain from (4.24) that

$$
\Lambda_{j}^{(1)} \leq \Lambda_{u, j}^{(1)} \leq \sqrt{2 \pi} \cos \frac{(2 j-1) \pi}{4 N}
$$

and the upper bound $\Lambda_{u, j}^{(1)}$ of $\Lambda_{j}^{(1)}$ is monotonically decreasing in $j$. At the same time, calculations indicate the behavior of $\Lambda_{j}^{(1)}$ given by Table 1. Our hypothesis is that each $\Lambda_{j}^{(1)}$ is also monotonically decreasing in $j$ and $\Lambda_{u, j}^{(1)}=2 \cos \frac{(2 j-1) \pi}{4 N}$.

TABLE 1. The behavior of $\Lambda_{j}^{(1)}$ and of $\Lambda_{u, j}^{(1)}$ for $N=8$.

\begin{tabular}{|c|c|c|c|c|c|c|c|c|}
\hline$j$ & 1 & 2 & 3 & 4 & 5 & 6 & 7 & 8 \\
\hline$\Lambda_{j}$ & 1.9807 & 1.8516 & 1.6037 & 1.2630 & .8934 & .5213 & .2462 & $.3097 e-1$ \\
\hline$\Lambda_{u, j}$ & 1.9903 & 1.9138 & 1.76384 & 1.5460 & 1.2687 & .9427 & .5805 & .1960 \\
\hline
\end{tabular}

Corollary 4.3. The numbers $\Lambda_{j}^{(1)}$ remain bounded also if $\chi_{j}(\eta)=e^{-z\left(x_{j, N}-\eta\right)}$, where $z=\rho e^{i \theta}$ is a complex number with $\rho \geq 0, \theta \in(-\pi / 2, \pi / 2)$.

Actually, we have in this case

$$
\begin{aligned}
& \Lambda_{j}^{(1)}=\sum_{k=1}^{N}\left|\int_{-1}^{x_{j, N}} e^{-\exp \{i \theta\}\left(x_{j, N}-\eta\right)} L_{k, N-1}(\eta) d \eta\right| \\
& \leq \sum_{k=1}^{N}\left|\int_{-1}^{x_{j, N}} \chi_{j, 1}(\eta) L_{k, N-1}(\eta) d \eta\right| \\
& +\sum_{k=1}^{N}\left|\int_{-1}^{x_{j, N}} \chi_{j, 2}(\eta) L_{k, N-1}(\eta) d \eta\right|
\end{aligned}
$$

where

$$
\begin{aligned}
& \chi_{j, 1}(\eta)=e^{-\rho \cos \theta\left(x_{j, N}-\eta\right)} \cos \left[\rho \sin \theta\left(x_{j, N}-\eta\right)\right], \\
& \chi_{j, 2}(\eta)=e^{-\rho \cos \theta\left(x_{j, N}-\eta\right)} \sin \left[\rho \sin \theta\left(x_{j, N}-\eta\right)\right] .
\end{aligned}
$$

Applying Lemma 4.1 for each summand and each of functions $\chi_{j, 1}(\eta) \leq 1$ and $\chi_{j, 1}(\eta) \leq 1$ we arrive at the estimate

$$
\Lambda_{j}^{(1)} \leq 2 \sqrt{2 \pi} \cos \frac{(2 j-1) \pi}{4 N}, j=1, \ldots, N .
$$

Now, we are in the position to estimate $\psi_{j}^{(3)}$.

To this end we assume that

(iii) The function $f(t, y)=f(t, y ; N)$ in the domain

$$
G=\left\{(t, y, N): 0 \leq t \leq 1,\left|\|y-u \mid\|<\gamma, N \geq N_{0}\right\},\right.
$$

in addition to (i), (ii), satisfies

$$
\left|\left\|A ^ { \alpha } [ f ( t , y _ { 1 } ) - f ( t , y _ { 2 } ) ] \left|\left\|\leq L\left|\left\|y_{1}-y_{2} \mid\right\| \forall y_{1}, y_{2} \in G,\right.\right.\right.\right.\right.
$$


for all $\left(t, y_{i}, N\right) \in G, i=1,2$, where $|\|Z\||=|\|y-u\||=\max _{j=1, \ldots, N}\left\|y_{j}-u\left(t_{j}\right)\right\|$, $\gamma$ is a positive real constant and $N_{0}$ is a fixed natural number large enough.

Under this assumption and taking into account Lemma 4.1 and Corollary 4.3 as well as (4.11), (4.12), we have

$$
\begin{aligned}
& \left\|\psi_{j}^{(3)}\right\| \leq c L h \sum_{k=-N_{1}}^{N_{1}} \frac{\left|z^{\prime}(k h)\right|}{|z(k h)|^{1+\alpha}} \sum_{l=1}^{N}\left|\int_{-1}^{x_{j, N}} e^{-z(k h)\left(x_{j, N}-\eta\right)} L_{l, N-1}(\eta) d \eta\right|\left\|Z_{l}\right\| \\
& \leq|\|Z\|| c L h \sum_{k=-N_{1}}^{N_{1}} \frac{\left|z^{\prime}(k h)\right|}{|z(k h)|^{1+\alpha}} \Lambda_{j}^{(1)} \leq c L S_{N_{1}} \Lambda_{j}^{(1)}|\|Z\|| \leq \frac{c^{*}}{\alpha} L|\|Z\||
\end{aligned}
$$

with a new positive constant $c^{*}$. This estimate together with (4.2) implies

$$
|\|Z\|| \leq \frac{\alpha}{\alpha-c^{*} L}|\|\psi\||
$$

with a constant $c$ independent of $\alpha, N$ provided that $c^{*} L / \alpha<1$. Analogously we obtain

$$
\left\|A^{\alpha} \psi_{j}^{(3)}\right\| \leq \frac{c^{*}}{\beta-\alpha} L\left|\left\|A^{\beta} Z\right\|\right| \quad \forall \beta>\alpha>0
$$

and

$$
\left|\left\|A ^ { \alpha } Z \left|\left\|\leq \frac{\beta-\alpha}{\beta-\alpha-c^{*} L}\left|\left\|A^{\beta} \psi\right\|\right| \quad \forall \beta>\alpha>0\right.\right.\right.\right.
$$

with a constant $c^{*}$ independent of $\alpha, \beta, N$ provided that $c^{*} L /(\beta-\alpha)<1$.

Taking into account (4.2) and estimates (4.5), (4.8), (4.14) as well as (4.30) we arrive at the estimate

$$
\begin{array}{r}
|\|Z\|| \leq \frac{c}{\alpha-c^{*} L} \times\left(e^{-c_{1} \sqrt{N_{1}}}\left(\left\|A^{\alpha} u_{0}\right\|+\int_{0}^{1}\left\|A^{\alpha} f(t, u(t))\right\| d t\right)\right. \\
\left.+\ln N \rho^{-N} \sup _{z \in D_{\rho}}\left\|A^{\alpha} f(z, u(z))\right\|\right)
\end{array}
$$

provided that the Lipschitz constant $L$ is such that $c^{*} L / \alpha<1$. Equalizing the exponents by $N \asymp \sqrt{N_{1}}$ (i.e. the number of the interpolation points must be proportional to the square root of the number of nodes in the Sinc-quadrature) we obtain

$$
\begin{aligned}
& |\|Z\||=|\|u-y\|| \leq \frac{c}{\alpha-c^{*} L} \ln N_{1} e^{-c_{1} \sqrt{N_{1}}} \\
& \times\left(\left\|A^{\alpha} u_{0}\right\|+\int_{0}^{1}\left\|A^{\alpha} f(t, u(t))\right\| d t+\sup _{z \in D_{\rho}}\left\|A^{\alpha} f(z, u(z))\right\|\right),
\end{aligned}
$$

and due to (4.6), (4.9), (4.15), (4.33) in a more strong norm

$$
\begin{aligned}
& \left|\left\|A^{\alpha} Z\right\|\right|=\left|\left\|A^{\alpha}(u-y)\right\|\right| \leq \frac{c}{\beta-\alpha-c^{*} L} \ln N_{1} e^{-c_{1} \sqrt{N_{1}}} \\
& \times\left(\left\|A^{\beta} u_{0}\right\|+\int_{0}^{1}\left\|A^{\beta} f(t, u(t))\right\| d t+\sup _{z \in D_{\rho}}\left\|A^{\beta} f(z, u(z))\right\|\right), \\
& \forall \beta>\alpha>0, \beta-\alpha>c^{*} L .
\end{aligned}
$$


These estimates show in particular that the operator $\mathcal{A}$ is contractive on $G$ provided that $c^{*} L / \alpha<1$ and $N \geq N_{0}$. Taking into account the Banach fixed point theorem we obtain by usual arguments that there exists the unique solution of (3.19) in $G$ for which the estimate (4.35) holds.

Thus, we have proven the following main result of this paper.

Theorem 4.4. Let $A$ be a densely defined, closed, strongly positive linear operator with the domain $D(A)$ in a Banach space $X$ and let the assumptions (i), (ii), (iii) hold. Then algorithm $\mathbf{A} 1$ defined by (3.18) for the numerical solution of the nonlinear problem (1.1) possesses a uniform with respect to $t$ exponential convergence rate with estimates (4.35), (4.36) provided that $N \asymp \sqrt{N_{1}}$ and the Lipschitz constant $L$ is sufficiently small.

Remark 4.5. The same result can be obtained if one uses the interpolation polynomial on the Chebyshev-Gauss-Lobatto grid

$$
\omega_{N}^{C G L}=\left\{x_{k, N}=x_{k, N}^{C G L}=\cos \frac{(N-j) \pi}{N}, k=0,1, \ldots, N\right\}
$$

where the nodes are zeros of the polynomial $\left(1-x^{2}\right) T_{N}^{\prime}(x)$.

Example 4.6. In order to have a view of the possible size of the Lipschitz constant let us consider the following nonlinear Cauchy problem:

$$
\begin{aligned}
& \frac{d \vec{u}(t)}{d t}+A \vec{u}(t)=\vec{f}(t, \vec{u}(t)), t>0, \\
& \vec{u}(0)=\vec{u}_{0}
\end{aligned}
$$

with a linear self-adjoint positive definite operator $A$ such that

$$
A=A^{*} \geq \lambda_{0} I, \lambda_{0}>0 .
$$

In this case algorithm (3.18) takes the form

$$
\begin{aligned}
& \vec{y}\left(t_{j}\right)=\vec{y}_{j}=e^{-A t_{j}} \vec{u}_{0}+\sum_{p=1}^{N} \frac{1}{2} \int_{-1}^{x_{j, N}} e^{-A\left(x_{j, N}-\eta\right) / 2} L_{p, N-1}(\eta) d \eta \vec{f}\left(t_{p}, \vec{y}_{p}\right), \\
& j=1, \ldots, N
\end{aligned}
$$

For the error $\vec{z}_{j}=\vec{y}_{j}-\vec{u}\left(t_{j}\right)=\vec{y}_{j}-\vec{u}_{j}$ we have the equation

$$
\begin{aligned}
& \vec{z}_{j}=\sum_{p=1}^{N} \frac{1}{2} \int_{-1}^{x_{j, N}} e^{-A\left(x_{j, N}-\eta\right) / 2} L_{p, N-1}(\eta) d \eta\left[\vec{f}\left(t_{p}, \vec{y}_{p}\right)-\vec{f}\left(t_{p}, \vec{u}_{p}\right)\right]+\vec{\psi}_{j} \\
& j=1, \ldots, N
\end{aligned}
$$

where

$$
\vec{\psi}_{j}=\frac{1}{2} \int_{-1}^{x_{j, N}-\eta} e^{-A\left(x_{j, N}-\eta\right) / 2}\left[P_{N-1}(\eta, \vec{u})-\vec{f}\left(\frac{\eta+1}{2}, u\left(\frac{\eta+1}{2}\right)\right)\right] d \eta
$$


is the truncation error. Using the equation

$$
\begin{aligned}
& \int_{-1}^{x_{j, N}} e^{-A\left(x_{j, N}-\eta\right) / 2} L_{p, N-1}(\eta) d \eta \\
& =-\int_{-1}^{x_{j, N}} e^{-A\left(x_{j, N}-\eta\right) / 2} \frac{d}{d \eta} \int_{\eta}^{x_{j, N}} L_{p, N-1}(\xi) d \xi d \eta \\
& =e^{-A\left(x_{j, N}+1\right) / 2} \int_{-1}^{x_{j, N}} L_{p, N-1}(\eta) d \eta \\
& +\frac{1}{2} \int_{-1}^{x_{j, N}} A e^{-A\left(x_{j, N}-\eta\right) / 2} \int_{\eta}^{x_{j, N}} L_{p, N-1}(\xi) d \xi d \eta
\end{aligned}
$$

we obtain

$$
\begin{aligned}
& \vec{z}_{j}=\sum_{p=1}^{N} \frac{1}{2}\left\{e^{-A\left(x_{j, N}+1\right) / 2} \int_{-1}^{x_{j, N}} L_{p, N-1}(\eta) d \eta\right. \\
& \left.+\frac{1}{2} \int_{-1}^{x_{j, N}} A e^{-A\left(x_{j, N}-\eta\right) / 2} \int_{\eta}^{x_{j, N}} L_{p, N-1}(\xi) d \xi d \eta\right\}\left[\vec{f}\left(t_{p}, \overrightarrow{y_{p}}\right)-\vec{f}\left(t_{p}, \vec{u}_{p}\right)\right] \\
& +\vec{\psi}_{j}, \quad j=1, \ldots, N .
\end{aligned}
$$

Since $A$ is a self-adjoint, positive definite operator we have

$$
\begin{aligned}
& \left\|A e^{-A\left(x_{j, N}-\eta\right) / 2}\right\|=\max _{\lambda_{0} \leq \lambda<\infty}\left(\lambda e^{-\lambda\left(x_{j, N}-\eta\right) / 2}\right) \leq \frac{2}{e\left(x_{j, N}-\eta\right)}, \\
& \left\|e^{-A\left(x_{j, N}+1\right) / 2}\right\| \leq 1 .
\end{aligned}
$$

This estimate together with (4.44) and Lemma 4.1 implies

$$
\begin{aligned}
& \left\|\vec{z}_{j}\right\| \leq L \sum_{p=1}^{N} \frac{1}{2}\left|\int_{-1}^{x_{j, N}} L_{p, N-1}(\eta) d \eta\right||\|\vec{z}\|| \\
& +L \frac{1}{2} \sum_{p=1}^{N} \frac{1}{2} \int_{-1}^{x_{j, N}}\left\|A e^{-A\left(x_{j, N}-\eta\right) / 2}\right\| \cdot\left|\int_{-1}^{\eta} L_{p, N}(\xi) d \xi\right| d \eta \cdot|\|\vec{z}\||+\left\|\vec{\psi}_{j}\right\| \\
& \leq \frac{1}{2} L \Lambda_{j}^{(1)}\left(x_{j, N}\right)|\|\vec{z}\||+\frac{L}{2 e} \int_{-1}^{x_{j, N}} \frac{1}{x_{j, N}-\eta} \Lambda_{j}^{(2)}(\eta)|\|\vec{z}\||+\left\|\vec{\psi}_{j}\right\| \\
& \leq \frac{L}{2} \Lambda_{j}|\|\vec{z}\||+\frac{L \sqrt{\pi}}{2 e} \int_{-1}^{x_{j, N}} \frac{1}{\sqrt{x_{j, N}-\eta}}|\|\vec{z}\||+\left\|\vec{\psi}_{j}\right\| \\
& \leq\left(\sqrt{\frac{\pi}{2}}+\frac{\sqrt{2 \pi}}{e}\right) L|\|\vec{z}\||+\left\|\vec{\psi}_{j}\right\| .
\end{aligned}
$$

The last inequality yields the following condition:

$$
L<\frac{\sqrt{2} e}{\sqrt{\pi} e+2 \sqrt{\pi}}
$$

on the Lipschitz constant $L$ which provides the convergence of the fixed point iteration and the corresponding a priori estimate for $\|\vec{z}\| \mid$.

Remark 4.7. Given $N_{1}$ choose the integer number $N_{2}=\left[\sqrt{N_{1}}\right]$ and set $y(t)=$ $P_{N_{2}-1}(2 t-1 ; y)$ with $y$ defined by algorithm (3.18). In order to get an error 
estimate for all $t \in[0,1]$ we represent

$$
\begin{aligned}
Z(t) & =u(t)-P_{N_{2}-1}(2 t-1 ; y) \\
& =u(t)-P_{N_{2}-1}(2 t-1 ; u)+\left[P_{N_{2}-1}(2 t-1 ; u)-P_{N_{2}-1}(2 t-1 ; y)\right] .
\end{aligned}
$$

Taking into account that the Lebesque constant relating to the Chebyshev interpolation nodes is bounded by $c \ln N_{2}$ and using the estimates (4.35), $\| P_{N_{2}-1}(2 t-1 ; u)-$ $P_{N_{2}-1}(2 t-1 ; y)\left\|\leq c \ln N_{2}|\|Z\|| \leq c \ln ^{2} N_{2} e^{-c_{1} \sqrt{N_{2}}}\right.$ and $\| u(t)-P_{N_{2}-1}(2 t-1 ; u) \| \leq$ $c \ln N_{2} e^{-c_{1} \sqrt{N_{2}}}$, we derive

$$
\max _{0 \leq t \leq 1}\left\|u(t)-P_{N_{2}-1}(2 t-1 ; y)\right\| \leq c \ln ^{2} N_{2} e^{-c_{1} \sqrt{N_{2}}} .
$$

\section{Modified ALGORIthm FOR ARBitrary Lipschitz CONSTANT}

In this section we show how the algorithm above can be modified for a nonlinearity with an arbitrary Lipschitz constant. To this end we suppose that $u(t) \in$ $D\left(A^{\sigma}\right), \sigma>c^{*} L / 2$. We cover the interval $[0,1]$ by the grid $\omega_{G}=\left\{t_{i}=i\right.$. $\tau: i=0,1, \ldots, K, \tau=1 / K\}$ and consider problem (1.1) on each subinterval $\left[t_{k-1}, t_{k}\right], k=1, \ldots, K$. The substitution $t=t_{k-1}(1-\xi) / 2+t_{k}(1+\xi) / 2, v(\xi)=$ $u\left(t_{k-1}(1-\xi) / 2+t_{k}(1+\xi) / 2\right)$ translates the original equation into the differential equation

$$
v^{\prime}(\xi)+\tilde{A} v=\tilde{f}(\xi, v)
$$

on the reference interval $[-1,1]$ with $\tilde{A}=\frac{\tau}{2} A$ and with the function $\tilde{f}(\xi, v)=$ $\frac{\tau}{2} f\left(t_{k-1}(1-\xi) / 2+t_{k}(1+\xi) / 2, u\left(t_{k-1}(1-\xi) / 2+t_{k}(1+\xi) / 2\right)\right)$ satisfying the Lipschitz condition with the Lipschitz constant $\tilde{L}=\tau L / 2$ which can be made arbitrarily small by the appropriate choice of $\tau$. We cover each subinterval $\left[t_{k-1}, t_{k}\right]$ by the Chebyshev-Gauss-Lobatto grid

$$
\begin{aligned}
& \omega_{k, N}^{C G L}=\left\{t_{k, j}: t_{k, j}=t_{k-1}\left(1-x_{j, N}\right) / 2+t_{k}\left(1+x_{j, N}\right) / 2, j=0,1, \ldots, N\right\} \\
& x_{j, N}=\cos (\pi(N-j) / N)
\end{aligned}
$$

and denote $v_{k}\left(x_{j, N}\right)=v_{k, j}=u\left(t_{k, j}\right), v_{k, 0}=v_{k}, u\left(t_{k, 0}\right)=u\left(t_{k}\right)=u_{k}, \vec{v}_{k}=$ $\left[v_{k, j}\right]_{j=1, \ldots, N}, \vec{u}_{k}=\left[u\left(t_{k, j}\right)\right]_{j=1, \ldots, N}$. Then, algorithm (3.18) with the corresponding Chebyshev-Gauss-Lobatto interpolation polynomial can be applied which provides an exponential accuracy on the subinterval $\left[t_{k-1}, t_{k}\right]$ under the assumption that the initial vector $u_{k-1}$ is known. This is exactly the case for $k=1$, and by algorithm (3.18) we obtain a value $v_{1, N}=v_{1}$ as an approximation for $u\left(t_{1}\right)$. Starting on the subinterval $\left[t_{1}, t_{2}\right]$ with the approximate initial value $v_{1}$ we obtain an approximate solution for this subinterval and so on.

In order to write down this idea as an algorithm we derive from (5.1) the relation

$$
v_{k, j}=e^{-\tilde{A}\left(1+x_{j, N}\right)} u_{k-1}+\int_{-1}^{x_{j, N}} e^{-\tilde{A}\left(x_{j, N}-\eta\right)} \tilde{f}\left(\eta, v_{k}(\eta)\right) d \eta .
$$

Denoting by $y_{k, j}$ approximations to $v_{k, j}$, approximating the operator exponential by (2.22) with $N_{1}$ nodes and the nonlinearity by the Chebyshev-Gauss-Lobatto 
interpolation polynomial

$$
\begin{aligned}
& P_{N}(\eta, \overrightarrow{\tilde{f}})=\sum_{l=0}^{N} \tilde{f}\left(x_{l, N}, y_{k, l}\right) L_{l, N}(\eta), \\
& L_{l, N}(\eta)=\frac{\left(1-\eta^{2}\right) T_{N}^{\prime}(\eta)}{\left(\eta-x_{l, N}\right) \frac{d}{d \eta}\left[\left(1-\eta^{2}\right) T_{N}^{\prime}(\eta)\right]_{\eta=x_{l, N}}}, \\
& \overrightarrow{\tilde{f}}=\left[\tilde{f}\left(x_{j, N}, y_{k, j}\right)\right]_{j=0}^{N}
\end{aligned}
$$

we arrive at the following system of nonlinear equations (analogous to (3.18)):

$$
y_{k, j}=e_{N_{1}}^{-\tilde{A}\left(1+x_{j, N}\right)} y_{k-1}+\int_{-1}^{x_{j, N}} e_{N_{1}}^{-\tilde{A}\left(x_{j, N}-\eta\right)} P_{N}(\eta, \overrightarrow{\tilde{f}}) d \eta
$$

which expresses $y_{k, j}, j=1,2, \ldots, N$ (in particular $y_{k, N}=y_{k+1}$ ) through $y_{k-1}$.

Now, we can formulate the following algorithm.

\section{Algorithm A2.}

Given $K$ satisfying (5.9), $N_{1}$ computes the approximate solution of nonlinear problem (1.1) with an arbitrary Lipschitz constant by solving of the nonlinear discrete system (5.5) on each subinterval

1. Choose $K$ satisfying (5.9) and $N_{1}$, and set $\tau=1 / K, t_{0}=0, y_{0}=u_{0}$.

2. For $i:=1$ step 1 to $K$ do

2.1. Set $t_{i}=t_{i-1}+\tau$ and find the approximate solution $y_{i, j}, j=1,2, \ldots, N$, of problem (1.1) on the Chebyshev-Gauss-Lobatto grid (5.2) covering the interval $\left[t_{i-1}, t_{i}\right]$ by algorithm (5.5) using $y_{i-1}$ as the initial value.

2.2. Set $y_{i}=y_{i, N}$.

Now, let us analyze the error $z_{k, j}=u\left(t_{k, j}-y_{k, j}\right)=v_{k, j}-y_{k, j}$ of this algorithm. We have the representation

$$
z_{k, j}=\psi_{k, j}+\sum_{p=0}^{3} \psi_{k, j}^{(p)}
$$

where

$$
\begin{aligned}
\psi_{k, j} & =e_{N_{1}}^{-\tilde{A}\left(x_{j, N}+1\right)} z_{k-1}, \\
\psi_{k, j}^{(0)} & =\left[e^{-\tilde{A}\left(x_{j, N}+1\right)}-e_{N_{1}}^{-\tilde{A}\left(x_{j, N}+1\right)}\right] u_{k-1}, \\
\psi_{k, j}^{(1)} & =\int_{-1}^{x_{j, N}}\left[e^{-\tilde{A}\left(x_{j, N}-\eta\right)}-e_{N_{1}}^{-\tilde{A}\left(x_{j, N}-\eta\right)}\right] \tilde{f}\left(\eta, v_{k}(\eta)\right) d \eta, \\
\psi_{k, j}^{(2)} & =\int_{-1}^{x_{j, N}} e_{N_{1}}^{-\tilde{A}\left(x_{j, N}-\eta\right)}\left[\tilde{f}\left(\eta, v_{k}(\eta)\right)-\sum_{l=0}^{N} \tilde{f}\left(x_{l, N}, v_{k, l}\right) L_{l, N}(\eta)\right] d \eta, \\
\psi_{k, j}^{(3)} & =\int_{-1}^{x_{j, N}} e_{N_{1}}^{-\tilde{A}\left(x_{j, N}-\eta\right)} \sum_{l=0}^{N}\left[\tilde{f}\left(x_{l, N}, v_{k, l}\right)-\tilde{f}\left(x_{l, N}, y_{k, l}\right)\right] L_{l, N}(\eta) d \eta .
\end{aligned}
$$


Under the same assumptions and analogously to (4.5), (4.8), (4.14), (4.30) we obtain the following estimates:

$$
\begin{aligned}
\left\|\psi_{k, j}\right\| & \leq \frac{c}{\alpha_{k}}\left\|\tilde{A}^{\alpha_{k}} z_{k-1}\right\|, \\
\left\|\psi_{k, j}^{(0)}\right\| & \leq \frac{c}{\alpha_{k}} \exp \left(-\sqrt{\frac{\pi d \alpha_{k}}{2}\left(N_{1}+1\right)}\right)\left\|\tilde{A}^{\alpha_{k}} u_{k-1}\right\|, \\
\left\|\psi_{k, j}^{(1)}\right\| & \leq \frac{c}{\alpha_{k}} \exp \left(-\sqrt{\frac{\pi d \alpha_{k}}{2}\left(N_{1}+1\right)}\right) \int_{-1}^{1}\left\|\tilde{A}^{\alpha_{k}} \tilde{f}\left(t, v_{k}(t)\right)\right\| d t \\
\left\|\psi_{k, j}^{(2)}\right\| & \leq \frac{c}{\alpha_{k}} \ln N \rho^{-N} \sup _{z \in D_{\rho_{k}}}\left\|\tilde{A}^{\alpha_{k}} \tilde{f}\left(z, v_{k}(z)\right)\right\|, \\
\left\|\psi_{k, j}^{(3)}\right\| & \leq \frac{c^{*}}{\alpha_{k}} \frac{L \tau}{2}\left\|\vec{z}_{k}\right\| \|,
\end{aligned}
$$

where $\alpha_{k}$ are some positive numbers, $D_{\rho_{k}}$ are the analyticity ellipses for $\tilde{A}^{\alpha_{k}} \tilde{f}\left(z, v_{k}(z)\right)$ and $\left\|\mid \vec{z}_{k}\right\|\left\|=\max _{1 \leq j \leq N}\right\| z_{k, j} \|$. Choosing $\tau=1 / K$ such that

$$
\frac{c^{*}}{\alpha_{k}} \frac{L \tau}{2}<1
$$

we obtain from (5.6), (5.8)

$$
\begin{aligned}
& \left\|\left|\vec{z}_{k}\right|\right\|=\max _{1 \leq j \leq N}\left\|z_{k, j}\right\| \leq \frac{c(\tau / 2)^{\alpha_{k}}}{\alpha_{k}-c^{*} L \tau / 2}\left\{\left\|A^{\alpha_{k}} z_{k-1}\right\|\right. \\
& +\left[\int_{t_{k-1}}^{t_{k}}\left\|A^{\alpha_{k}} f(t, u(t))\right\| d t+\left\|A^{\alpha_{k}} u\left(t_{k-1}\right)\right\|\right] \exp \left(-\sqrt{\frac{\pi d \alpha_{k}}{2}}\left(N_{1}+1\right)\right) \\
& \left.+\frac{\tau}{2} \ln N \rho^{-N} \sup _{z \in D_{\rho_{k}}}\left\|A^{\alpha_{k}} f\left(t_{k}(z), u\left(t_{k}(z)\right)\right)\right\|\right\}, \\
& k=2,3, \ldots, K, \\
& t_{k}(z)=t_{k-1} \frac{1-z}{2}+t_{k} \frac{1+z}{2} .
\end{aligned}
$$

Equalizing the exponents by setting $N \asymp \sqrt{N_{1}}$ (i.e. the number of the interpolation points on each subinterval must be proportional to the square root of the number of nodes in the Sinc approximation of the operator exponential) we obtain from (5.10)

$$
\begin{aligned}
& \left\|\left|A^{\alpha_{k+1}} \vec{z}_{k}\right|\right\| \leq \frac{c(\tau / 2)^{\alpha_{k}}}{\alpha_{k}-\alpha_{k+1}-c^{*} L \tau / 2}\left\{\left|\left\|A^{\alpha_{k}} z_{k-1} \mid\right\|\right.\right. \\
& +\ln N_{1} e^{-c_{1} \sqrt{N_{1}}}\left[\int_{t_{k-1}}^{t_{k}}\left\|A^{\alpha_{k}} f(t, u(t))\right\| d t+\left\|A^{\alpha_{k}} u\left(t_{k-1}\right)\right\|\right. \\
& \left.\left.+\frac{\tau}{2} \sup _{z \in D_{\rho_{k}}}\left\|A^{\alpha_{k}} f\left(t_{k}(z), u\left(t_{k}(z)\right)\right)\right\|\right]\right\}, \\
& k=2,3, \ldots, K
\end{aligned}
$$


where $\alpha_{k}$ satisfy

$$
\begin{aligned}
& \alpha_{k}-\alpha_{k+1}-c^{*} L \tau / 2>0, \\
& 0<\alpha_{k} \leq \sigma, \quad k=1,2, \ldots, K .
\end{aligned}
$$

Taking into account that $z_{0}=0$ we have for $k=1$ the estimate

$$
\begin{aligned}
& \quad\left\|A^{\alpha_{2}} \vec{z}_{k}\right\| \mid \leq \frac{c(\tau / 2)^{\alpha_{1}}}{\alpha_{1}-\alpha_{2}-c^{*} L \tau / 2} \ln N_{1} e^{-c_{1} \sqrt{N_{1}}}\left[\left\|A^{\alpha_{1}} u_{0}\right\|+\int_{t_{0}}^{t_{1}}\left\|A^{\alpha_{1}} f(t, u(t))\right\| d t\right. \\
& \left.\quad+\frac{\tau}{2} \sup _{z \in D_{\rho_{1}}}\left\|A^{\alpha_{k}} f\left(t_{k}(z), u\left(t_{k}(z)\right)\right)\right\|\right] .
\end{aligned}
$$

Estimate (5.11) can be rewritten in the form

$$
w_{k} \leq \mu_{k}\left(g_{k}+w_{k-1}\right), \quad k=1,2, \ldots, K,
$$

with

$$
\begin{aligned}
& w_{k}=\left\|\left|\left\|A^{\alpha_{2}} \vec{z}_{k}\right\|\right|, \quad \mu_{k}=\frac{c(\tau / 2)^{\alpha_{1}}}{\alpha_{k}-\alpha_{k+1}-c^{*} L \tau / 2},\right. \\
& g_{k}=\ln N_{1} e^{-c_{1} \sqrt{N_{1}}}\left[\int_{t_{k-1}}^{t_{k}}\left\|A^{\alpha_{k}} f(t, u(t))\right\| d t+\left\|A^{\alpha_{k}} u\left(t_{k-1}\right)\right\|\right. \\
& \left.+\frac{\tau}{2} \sup _{z \in D_{\rho_{k}}}\left\|A^{\alpha_{k}} f\left(t_{k}(z), u\left(t_{k}(z)\right)\right)\right\|\right]
\end{aligned}
$$

which yields

$$
w_{k} \leq \mu_{k}\left(g_{k}+w_{k-1}\right), \quad k=1,2, \ldots, K,
$$

and further recursively

$$
w_{k} \leq \mu_{k} g_{k}+\mu_{k-1} \mu_{k} g_{k-1}+\cdots+\mu_{1} \mu_{2} \cdots \mu_{k} g_{1} .
$$

Conditions (5.12) imply

$$
0<\alpha_{k+1}<\alpha_{1}-c^{*} L k \tau / 2>0, \quad k=1,2, \ldots, K .
$$

Let us choose $\alpha_{1}=\sigma, \varepsilon \in\left(0, \frac{\sigma-c^{*} L / 2}{c^{*} L}\right)$ and

$$
\alpha_{k+1}=\sigma-\left(\frac{1}{2}+\varepsilon\right) c^{*} L k \tau, \quad k=1,2, \ldots, K .
$$

Then we have

$$
\rho_{k}=\frac{c(\tau / 2)^{\alpha_{k}}}{\varepsilon c^{*} L \tau}=\frac{c(\tau / 2)^{\alpha_{k}-1}}{2 \varepsilon c^{*} L}<\frac{c}{2 \varepsilon c^{*} L}(\tau / 2)^{\sigma-(0.5+\varepsilon) c^{*} L}=q
$$

and (5.17) implies

$$
\max _{1 \leq k \leq K} w_{k} \leq \max \left\{q^{K}, q\right\} \sum_{p=1}^{K} g_{p}
$$




$$
\begin{aligned}
\max _{1 \leq k \leq K}\left|\left\|A^{\alpha_{2}} \vec{z}_{k}\right\|\right| & \\
\leq \max \left\{q^{K}, q\right\} \ln N_{1} e^{-c_{1} \sqrt{N_{1}}} \sum_{k=1}^{K} & {\left[\int_{t_{k-1}}^{t_{k}}\left\|A^{\alpha_{k}} f(t, u(t))\right\| d t+\left\|A^{\alpha_{k}} u\left(t_{k-1}\right)\right\|\right.} \\
& \left.+\frac{\tau}{2} \sup _{z \in D_{\rho_{k}}}\left\|A^{\alpha_{k}} f\left(t_{k}(z), u\left(t_{k}(z)\right)\right)\right\|\right] .
\end{aligned}
$$

Thus, we have proven the following second main result of this paper concerning the rate of convergence of Algorithm A2.

Theorem 5.1. Let $A$ be a densely defined closed strongly positive linear operator with the domain $D(A)$ in a Banach space $X$ and let the assumptions (i), (ii), (iii) hold. If the solution of the nonlinear problem (1.1) belongs to the domain $D\left(A^{\sigma}\right)$ with $\sigma>c^{*} L / 2$, then Algorithm A2 possesses a uniform with respect to $t$ exponential convergence rate with estimate (5.22), provided that $N \asymp \sqrt{N_{1}}$ and the chosen number of subintervals $K$ satisfies (5.9).

\section{IMPLEMENTATION OF THE ALGORITHM}

Algorithm (3.18) represents a nonlinear system of algebraic equations which can be solved by the fixed point iteration

$$
\begin{aligned}
& y_{j}^{(m+1)}=\frac{h}{2 \pi i} \sum_{k=-N_{1}}^{N_{1}} \mathcal{F}_{h}\left(x_{j, N}, k h\right) \\
& +\frac{h}{4 \pi i} \int_{-1}^{x_{j}} \sum_{k=-N_{1}}^{N_{1}} F_{A}\left(\xi, x_{j}-\eta\right) P_{N-1}\left(\eta ; f\left(\cdot, y^{(m)}\right)\right) d \eta, \\
& j=1, \ldots, N, m=0,1, \ldots
\end{aligned}
$$

Since the operator $\mathcal{A}$ is contractive we obtain the following inequality:

$$
||\left|y_{j}^{(m+1)}-y_{j}^{(m)}\right||| \leq L c^{*}||\left|y_{j}^{(m)}-y_{j}^{(m-1)}\right|||
$$

which justifies the convergence of the fixed point iteration (6.1) with the speed of a geometric progression with the denominator $L c^{*}<1$, provided the assumptions of Theorem 4.4 hold.

Let us estimate the asymptotical computational costs of our method and a possible alternative polynomially convergent method (e.g. step-by-step implicit Euler method) in order to arrive at a given tolerance $\varepsilon$. Assuming the time step $\tau$ and the spatial step $h$ in the Euler scheme to be equal we have asymptotically to make $\frac{t^{*}}{\varepsilon}$ steps in order to arrive at a tolerance $\varepsilon$ at a given fixed point $t=t^{*}$. At each step the nonlinear equation $\tau f\left(t_{k+1}, y_{k+1}\right)-\left(\tau A y_{k+1}+I\right)=y_{k}$ should be solved, where $y_{k}$ is an approximation for $u\left(t_{k}\right)$. Assuming the computational costs for the solution of this nonlinear equation to be $M$ we arrive at the total computational costs for the Euler method $T_{E} \asymp t^{*} M / \varepsilon$. From the asymptotical relation $\ln N_{1} e^{-c \sqrt{N_{1}}}<e^{-c_{1} \sqrt{N_{1}}} \asymp \varepsilon$ we obtain that in our algorithm $N \asymp \sqrt{N_{1}} \asymp \ln (1 / \varepsilon)$. It is natural to assume that the computational costs for the numerical solution of the nonlinear equation (6.1) (or (3.19)) are not greater than $N M \asymp \sqrt{N_{1}} M$. Then the total costs of our algorithm are $T_{O} \asymp M \ln (1 / \varepsilon) \ll T_{E}$ for $\varepsilon$ small enough. 
Example 6.1. Let us consider the nonlinear initial value problem

$$
\begin{aligned}
& u^{\prime}(t)+u(t)=\mu e^{-2 t}-\mu[u(t)]^{2}, t \in(-1,1], \\
& u(-1)=e
\end{aligned}
$$

with the exact solution $u(t)=e^{-t}$ (independent of $\mu$ ). The equivalent Volterra integral equation is

$$
u(t)=\varphi(t)-\mu \int_{-1}^{t} e^{-(t-s)} u^{2}(s) d s
$$

where

$$
\varphi(t)=e^{-t}+\mu\left[e^{1-t}-e^{-2 t}\right] .
$$

Algorithm (3.18) combined with the fixed point iteration takes in this case the form

$$
\begin{aligned}
& y_{j}^{(m+1)}=\varphi_{j}-\mu \sum_{p=1}^{N} \alpha_{p, j}\left[y_{p}^{(m)}\right]^{2}, \\
& y_{j}^{(0)}=1 / 2, j=1, \ldots, N, m=0,1, \ldots,
\end{aligned}
$$

where

$$
\begin{aligned}
& \alpha_{p, j}=e^{-x_{j, N}} \int_{-1}^{x_{j, N}} L_{p, N-1}(s) e^{s} d s, \\
& y_{j}=y\left(x_{j, N}\right), \varphi_{j}=\varphi\left(x_{j, N}\right) .
\end{aligned}
$$

The algorithm was implemented in Maple 8 (Digits=30) for $\mu=1 / 4$ where integrals (6.7) were computed analytically. We denote by $I t$ the number of iterations necessary to satisfy the interruption criterium $\left|y_{J, N}^{(m+1)}-y_{J, N}^{(m)}\right|<e^{-N} \cdot 10^{-2}$ and accept $y_{j}^{(I t)}=y_{J, N}^{(m+1)}$ as the approximate solution. The error is computed as $\varepsilon_{N}=\|u-y\|_{N, \infty}=\max _{1 \leq j \leq N}\left|u\left(x_{j, N}\right)-y_{j}^{(I t)}\right|$. The numerical results are given by Table 2 and confirm our theory.

TABLE 2. The error of algorithm (3.18) for problem (6.3).

\begin{tabular}{|c|c|c|}
\hline$N$ & $\varepsilon_{N}$ & $I t$ \\
\hline 2 & 0.129406 & 6 \\
\hline 4 & $0.626486 \mathrm{e}-2$ & 8 \\
\hline 8 & $0.181353 \mathrm{e}-5$ & 9 \\
\hline 16 & $0.162597 \mathrm{e}-14$ & 16 \\
\hline 32 & $0.110000 \mathrm{e}-28$ & 26 \\
\hline
\end{tabular}

Example 6.2. Let us consider the problem

$$
\begin{aligned}
& \frac{\partial u}{\partial t}+A u=f(t, u(t)), \\
& u(-1)=u_{0}
\end{aligned}
$$

with the linear operator $A$ given by

$$
\begin{aligned}
& D(A)=\left\{w(x) \in H^{2}(0,1): w^{\prime}(0)=0, w^{\prime}(1)=0\right\}, \\
& A v=-w^{\prime \prime} \forall w \in D(A),
\end{aligned}
$$


with the nonlinear operator $f$ given by

$$
f(t, u)=-2 t u^{2}
$$

and with the initial condition given by

$$
u_{0}=u(-1, x)=1 / 2 .
$$

Since the numerical algorithm above supposes that the operator coefficient is strongly positive, we shift its spectrum by the variables transform $u(t, x)=e^{d^{2} t} v(t, x)$ with a real number $d$. Then we obtain the problem

$$
\begin{aligned}
& \frac{\partial v}{\partial t}+A_{d} v=f_{d}(t, v(t)), \\
& v(-1)=v_{0}
\end{aligned}
$$

with the linear operator $A_{d}$ given by

$$
\begin{aligned}
& D\left(A_{d}\right)=D(A), \\
& A_{d} w=A w+d^{2} w \forall w \in D\left(A_{d}\right),
\end{aligned}
$$

with the nonlinear operator $f_{d}$ given by

$$
f_{d}(t, v)=-2 t e^{d^{2} t} v^{2}
$$

and with the initial condition

$$
v_{0}=v(-1, x)=e^{d^{2}} / 2 .
$$

It is easy to check that the exact solution of this problem is

$$
v(t, x)=e^{-d^{2} t} /\left(1+t^{2}\right) .
$$

The equivalent Volterra integral equation for $v$ has the form

$$
v(t, x)=\frac{1}{2} e^{-A_{d}(t+1)} e^{d^{2}}-2 \int_{-1}^{t} e^{-A_{d}(t-s)} s e^{d^{2} s}[v(s, \cdot)]^{2} d s .
$$

Returning to the unknown function $u$ the integral equation takes the form

$$
u(t, x)=\frac{1}{2} e^{-A_{d}(t+1)} e^{d^{2}(t+1)}-2 \int_{-1}^{t} e^{-A_{d}(t-s)} s e^{-d^{2} s}[u(s, \cdot)]^{2} d s .
$$

Our algorithm was implemented in Maple with numerical results given by Table 3 where $\varepsilon_{N}=\max _{1 \leq j \leq N} \varepsilon_{j, N}, \varepsilon_{j, k, N}=\left|u\left(x_{j, N}, k h\right)-y_{j, k}\right|, j=1, \ldots, N, k=$ $-N_{1}, \ldots, N_{1}$. The numerical results are in good agreement with Theorem 4.4.

TABle 3. The error of algorithm (3.18) for problem (6.3).

\begin{tabular}{|c|c|c|}
\hline$N$ & $\varepsilon_{N}$ & $I t$ \\
\hline 4 & $0.8 \mathrm{e}-1$ & 12 \\
\hline 8 & $0.7 \mathrm{e}-3$ & 10 \\
\hline 16 & $0.5 \mathrm{e}-6$ & 11 \\
\hline 32 & $0.3 \mathrm{e}-12$ & 12 \\
\hline
\end{tabular}


Example 6.3. This example deals with the two-dimensional nonlinear problem

$$
\begin{aligned}
& \frac{\partial u}{\partial t}+A u=f(t, u(t)), \\
& u(0)=u_{0}
\end{aligned}
$$

where

$$
\begin{aligned}
& D(A)=\left\{w(x, y) \in H^{2}(\Omega):\left.w\right|_{\partial \Omega}=0\right\} \\
& A v=-\Delta v \forall v \in D(A), \\
& \Omega=[0,1] \times[0,1]
\end{aligned}
$$

with the nonlinear operator $f$ given by

$$
f(t, u)=-u^{3}+e^{-6 \pi^{2} t} \sin ^{3} \pi x \sin ^{3} \pi y
$$

and with the initial condition given by

$$
u_{0}=u(0, x, y)=\sin \pi x \sin \pi y .
$$

The exact solution is given by $u=e^{-2 \pi^{2} t} \sin \pi x \sin \pi y$. Algorithm (3.18) with $N=\sqrt{N_{1}}$ Chebyshev-Gauss-Lobatto nodes combined with the fixed point iteration provides the error which is presented in Table 4 .

TABLE 4. The error $\varepsilon_{N}$ of algorithm (3.18) for problem (6.19)-(6.22).

\begin{tabular}{|c|c|c|}
\hline$N$ & $\varepsilon_{N}$ & $I t$ \\
\hline 4 & $.3413 \mathrm{e}-6$ & 12 \\
\hline 8 & $.1761 \mathrm{e}-6$ & 10 \\
\hline 16 & $.8846 \mathrm{e}-7$ & 14 \\
\hline 32 & $.5441 \mathrm{e}-8$ & 14 \\
\hline
\end{tabular}

Example 6.4. Let us consider again the nonlinear initial value problem (6.3) and apply the Algorithm $\mathbf{A} 2$ for various values of the Lipschitz constant $2 \mu$. Inequality (4.47) guarantees the convergence of algorithm (3.18) combined with the fixed point iteration for $\mu<0.4596747673$. Numerical experiments indicate the convergence also for $\mu>0.4596747673$, but beginning with $\mu \approx 1$ the process becomes divergent and Algorithm A2 should be applied. The corresponding results for various $\mu$ are presented in Table 5 .

TABle 5. The results of Algorithm A2 for problem (6.3) with various values of the Lipschitz constant $\mu$.

\begin{tabular}{|c|c|c|}
\hline$\mu$ & $K$ & $I t$ \\
\hline 0.9 & 1 & 22 \\
\hline 1 & 2 & 20 \\
\hline 10 & 32 & 20 \\
\hline 20 & 50 & 25 \\
\hline 50 & 128 & 25 \\
\hline 100 & 256 & 24 \\
\hline
\end{tabular}


Here the degree of the interpolation polynomial is $N=16, K$ is the number of subintervals of the whole interval $[-1 ; 1]$, It denotes the number of the iterations in order to arrive at the accuracy $\exp (-N) * 0.01$.

\section{ACKNOWLEDGMENT}

The authors are grateful for the financial support made in part by Deutsche Forschungsgemeinschaft (DFG).

\section{REFERENCES}

[1] D.Z. Arov, I.P. Gavrilyuk and V.L. Makarov, Representation and approximation of solutions of initial value problems for differential equations in Hilbert space based on the Cayley transform, Progress in partial differential equations (C. Bandle et al. eds.), vol. 1, Pitman Res. Notes Math. Sci., 1995, pp. 40-50. MR.1416572 (97h:34074)

[2] A. Ashyralyev and P. Sobolevskii, Well-Posedness of Parabolic Difference Equations, Birkhäuser Verlag, Basel, 1994. MR.1299329 (95j:65094)

[3] N.Yu. Bakaev, Stability estimates for a general discretization method, Soviet Math. Dokl. 40 (1990), 11-15. MR1035844 (91e:65104)

[4] T.Ju. Bohonova, I.P. Gavrilyuk, V.L. Makarov and V. Vasylyk, Exponentially convergent Duhamel's like algorithms for differential equations with an operator coefficient possessing a variable domain in Banach space, Reports on Numerical Mathematics, Friedrich-SchillerUniversität Jena (http://www.minet.uni-jena.de/Math-Net/reports05/reports.html \#2005), 05-06 (2005), 1-25.

[5] C. Canuto, M.Y. Hussaini, A. Quarteroni and T.A. Zang, Spectral Methods in Fluid Dynamics, Springer-Verlag, Berlin, Heidelberg, New York et al., 1988. MR.917480 (89m:76004)

[6] M.L. Fernandez, Ch. Lubich, C. Palencia and A. Schädle, Fast Runge-Kutta approximation of inhomogeneous parabolic equations, Numerische Mathematik 5, (2005), 1-17.

[7] H. Fujita, N. Saito,and T. Suzuki, Operator Theory and Numerical Methods, Elsevier, Heidelberg, 2001.

[8] I.P. Gavrilyuk, Strongly P-positive operators and explicit representation of the solutions of initial value problems for second order differential equations in Banach space, Journ.of Math. Analysis and Appl. 1(88) (2003), 327-349. MR.1704587 (2001j:34072)

[9] I.P. Gavrilyuk, Algorithms without accuracy saturation and exponential convergent algorithms for operator equations, Journal of Numerical and Applied Mathematics (ISSN 08686912) 236 (1999), 28-43.

[10] I.P. Gavrilyuk, W. Hackbusch and B.N. Khoromskij, $\mathcal{H}$-matrix approximation for elliptic solution operators in cylinder domains, East-West Journal of Numerical Analysis 9 (2001), no. 1, 25-58. MR.1839197 (2002e:65064)

[11] I.P. Gavrilyuk, W. Hackbusch and B.N. Khoromskij, $\mathcal{H}$-matrix Approximation for the Operator Exponential with Applications, Numer. Math. 92 (2002), 83-111. MR1917366 (2003g:65061)

[12] I.P. Gavrilyuk, W. Hackbusch and B.N. Khoromskij, Data-sparse approximation to the operator-valued functions of elliptic operator, Math. Comp. 73 (2004), 1297-1324. MR2047088 (2005b:47086)

[13] I.P. Gavrilyuk, W. Hackbusch and B.N. Khoromskij, Hierarchical tensor-product approximation to the inverse and related operators for high-dimensional elliptic problems, Computing 74 (2005), 131-157. MR2133692 (2006f:65049)

[14] I.P. Gavrilyuk, W. Hackbusch and B.N. Khoromskij, Data-sparse approximation of a class of operator-valued functions, Math. Comp. 74 (2005), 681-708 MR2114643 (2005i:65068)

[15] I.P. Gavrilyuk and V.L. Makarov, Representation and approximation of the solution of an initial value problem for a first order differential eqation in Banach space, Z. Anal. Anwend. (ZAA) 15 (1996), 495-527. MR.1394440 (97h:65076)

[16] I.P. Gavrilyuk and V.L. Makarov, Algorithms without accuracy saturation for evolution equations in Hilbert and Banach spaces, Math. Comp. 74 (2005), 555-583. MR2114638 (2005j:65053) 
[17] I.P. Gavrilyuk and V.L. Makarov, Exponentially convergent parallel discretization methods for the first order evolution equations, Computational Methods in Applied Mathematics (CMAM) 1, 4, (2001), 333-355. MR.1892950(2003f:65174)

[18] I.P. Gavrilyuk and V.L. Makarov, The Cayley transform and the solution of an initial value problem for a first order differential equation with an unbounded operator coefficient in Hilbert space, Numer. Func. Anal. Optimiz. 15, (1994), 583-598. MR.1281563 (95b:34096)

[19] I.P. Gavrilyuk and V.L. Makarov, Exponentially convergent parallel discretization methods for the first order differential equations, Doklady of the Ukrainian Academy of Scienses 3, (2002), 1-6.

[20] I.P. Gavrilyuk and V.L. Makarov, Exponentially convergent algorithms for the operator exponential with applications to inhomogeneous problems in Banach spaces, SIAM J. Numer. Anal., 43(5):2144-2171, 2005. MR2192335 (2006m:65100)

[21] I.P. Gavrilyuk and V.L. Makarov, An explicit boundary integral representation of the solution of the two-dimensional heat equation and its discretization, J. Integral Equations Appl. 12, (Spring 2000), 1, 63-83. MR1760898(2001c:65129)

[22] I.P. Gavrilyuk and V.L. Makarov, An exponentially convergent algorithm for nonlinear differential equations in Banach spaces, Reports on Numerical Mathematics, Friedrich-ShillerUniversität Jena (http://www.minet.uni-jena.de/Math-Net/reports/) 02/05 (2005), 1-22.

[23] I.P. Gavrilyuk and V.L. Makarov and V. Vasylyk, A new estimate of the Sinc method for linear parabolic problems including the initial point, Computational Methods in Applied Mathematics (CMAM) 4, (2004), 2, 1-27. MR2119621 (2005m:65214)

[24] J.A. Goldstein, Semigroups of Linear Operators and Applications, Oxford University Press, New York, Clarendon Press, Oxford, 1985. MR790497 (87c:47056)

[25] D. González and C. Palencia, Stability of time-stepping methods for abstract time-dependent parabolic problems, SIAM J. Numer. Anal. 35, (2004), 3, 973-989. MR.1619918(99b:65072)

[26] D. Henry, Geometrical Theory of Semilinear Parabolic Equations, Springer-Verlag, BerlinHeidelberg-New York, 1981. MR610244 (83j:35084)

[27] M.A. Krasnosel'skij and P.E. Sobolevskij, Fractional powers of operators acting in Banach spaces (in Russian), Doklady AN SSSR 129, (1959), 3, 499-502. MR0108733 (21:7447)

[28] K. Kwon and D. Sheen, A parallel method for the numerical solution of integro-differential equation with positive memory, Comput. Methods Appl. Mech. Engrg. 192, (2003), 41-42, 4641-4658. MR2012483 (2004k:65263)

[29] M. López-Fernández, C. Palencia and A. Schädle, A spectral order method for inverting sectorial Laplace transforms, ZIB-Report 05-26, April 2005.

[30] M. López-Fernández and C. Palencia, On the numerical inversion of the Laplace transform of certain holomorphic mappings, Applied Numerical Mathematics, 51, (2004), 289-303. MR 2091405 (2005e:65210)

[31] M. López-Fernández, C. Palencia and A. Schädle, Fast Runge-Kutta approximation of inhomogeneous parabolic differential equations, Preprint 2005.

[32] J. Lund and K.L. Bowers, Sinc methods for quadrature and differential equations,SIAM, Philadelphia, 1992. MR1171217 (93i:65004)

[33] A. Pazy, Semigroups of linear operator and applications to partial differential equations, Springer-Verlag, Berlin-Heidelberg-New York, 1983. MR710486 (85g:47061)

[34] D. Sheen, I.H. Sloan and V. Thomée, A parallel method for time-discretization of parabolic problems based on contour integral representation and quadrature, Math.Comp. 69, (2000), 177-195. MR.1648403 (2000i:65161)

[35] D. Sheen,I.H. Sloan and V.Thomée, A parallel method for time-discretization of parabolic equations based on Laplace transformation and quadrature, IMA Journal of Numerical Analysis, 23, (2003), 269-299. MR 1975267 (2004b:65161)

[36] M.Z. Solomjak, Application of the semi-group theory to investigation of differential equations in Banach spaces (in Russian) , Doklady AN SSSR 122, (1958), 2, 766-769. MR0105029 $(21: 3775)$

[37] F. Stenger, Numerical methods based on Sinc and analytic functions. Springer Verlag, 1993. MR.1226236 (94k:65003)

[38] G. Szegö, Orthogonal Polynomials. American Mathematical Society,New York, 1959. MR0106295 (21:5029)

[39] G. Szegö, Orthogonal Polynomials (with an Introduction and a Complement by J.L. Geronimus), State Publishing House of Physical and Mathematical Literature, Moscow, 1962. 
[40] V. Thomée, A high order parallel method for time discretisation of parabolic type equations based on Laplace transformation and quadrature, Int. J. Numer Anal. Model. 2 (2005), 85-96. MR2112660 (2005i:65159)

[41] V. Vasylyk, Uniform exponentially convergent method for the first order evolution equation with unbounded operator coefficient, Journal of Numerical and Applied Mathematics (ISSN 0868-6912), 1, (2003), 99-104 (in Russian).

Staatliche Studienakademie Thueringen-Berufsakademie Eisenach, University of Cooperative Edukation, Am Wartenberg 2, D-99817 Eisenach, Germany

E-mail address: ipg@ba-eisenach.de

National Academy of Sciences of Ukraine, Institute of Mathematics, TereschenkivSKa 3, 01601 Kiev, UKRaine

E-mail address: makarov@imath.kiev.ua 